\title{
Review
}

\section{Functional interactions and signaling properties of mammalian DNA mismatch repair proteins}

\author{
A Bellacosa ${ }^{\star, 1,2}$ \\ 1 Human Genetics Program, Fox Chase Cancer Center, Philadelphia, PA 19111 \\ USA \\ 2 Department of Medical Genetics, Catholic University Medical School, 00168, \\ Rome, Italy \\ * Corresponding author: A Bellacosa, Human Genetics Program, Division of \\ Population Science, Fox Chase Cancer Center, 7701 Burholme Avenue, \\ Philadelphia, PA 19111, USA; E-mail: A_Bellacosa@fccc.edu
}

Received 9.11.00; revised 11.7.01; accepted 30.8.01

Edited by J Wang

\begin{abstract}
The mismatch repair (MMR) system promotes genomic fidelity by repairing base-base mismatches, insertion-deletion loops and heterologies generated during DNA replication and recombination. This function is critically dependent on the assembling of multimeric complexes involved in mismatch recognition and signal transduction to downstream repair events. In addition, MMR proteins coordinate a complex network of physical and functional interactions that mediate other DNA transactions, such as transcription-coupled repair, base excision repair and recombination. MMR proteins are also involved in activation of cell cycle checkpoint and induction of apoptosis when DNA damage overwhelms a critical threshold. For this reason, they play a role in cell death by alkylating agents and other chemotherapeutic drugs, including cisplatin. Inactivation of MMR genes in hereditary and sporadic cancer is associated with a mutator phenotype and inhibition of apoptosis. In the future, a deeper understanding of the molecular mechanisms and functional interactions of MMR proteins will lead to the development of more effective cancer prevention and treatment strategies. Cell Death and Differentiation (2001) 8, 1076- 1092.
\end{abstract}

Keywords: mismatch repair; DNA damage; apoptosis; signal transduction

Abbreviations: MMR, mismatch repair; IDL, insertion/deletion loop; MSI, microsatellite instability; MSS, microsatellite-stable; TCR, transcription-coupled repair; NER, nucleotide excision repair; GGR, global genome repair; BER, base excision repair; HNPCC, hereditary non-polyposis colorectal cancer

\section{Introduction}

Dealing with the potentially mutagenic consequences of DNA damage is a fundamental biological property acquired by living organisms early during evolution. Thus, it is not surprising that most mammalian DNA repair enzymes show significant structural similarities to their bacterial counterparts. However, mammalian DNA repair enzymes, like other mammalian proteins, often contain additional domains and structural features along with the catalytic region which directly processes DNA damage. It is fair to assume that these domains represent functional and regulatory add-on modules which provide interaction interfaces allowing coordination of DNA repair reactions with essential aspects of the biology of mammalian cells, such as cell cycle checkpoint control, transcription, DNA methylation, proliferation and apoptosis. Furthermore, through gene duplication during evolution and the modulation of splicing, multiple related DNA repair genes and different isoforms replace in eukaryotic cells the function of a single bacterial homologue. The combinatorial use of these multiple gene products increases exponentially the number of possible interactions; thus providing the necessary versatility in DNA damage recognition and coordination with other cellular events, which is appropriate for the complex metabolic circuitry of eukaryotic cells.

In this article, using the mammalian DNA mismatch repair (MMR) system as a case study, I will review the literature on the functional interactions and signaling pathways stemming from this specialized system of DNA repair.

\section{Interactions among mammalian DNA mismatch repair proteins}

The MMR system maintains genomic stability by repairing base-base mismatches and insertion/deletion loops (IDLs) originating during DNA replication, as well as heteroduplexes occurring during recombination. ${ }^{1-3}$ In so doing, MMR improves the fidelity of DNA biosynthesis by 100-1000-fold, complementing the intrinsic error-free and proofreading properties of replicative DNA polymerases and lowering the overall mutation rate to one error per $10^{10}$ nucleotides synthesized. $^{2}$

This role of genome stabilization is critically dependent on the ability of MMR proteins to recognize the DNA damage over a vast excess of normal DNA structures: in fact, it is estimated that a mismatch occurs every $10^{6}-10^{7}$ base pairs. ${ }^{4}$ In addition, a system is implemented, via the assembling of a signaling complex, to identify and discriminate the newly synthesized DNA strand, which by definition contains the misincorporated DNA base. The advantage of a signaling complex is that it can be adapted to perform additional tasks, such as the induction of apoptosis if DNA damage exceeds a given threshold (see below). Alterations in the accurate mechanisms of MMR have profound consequences, from an increase of the mutation rate in simple unicellular organisms ('mutator 
phenotype') to a pathogenetic role in hereditary and sporadic cancer in humans. ${ }^{5-8}$ The mechanistic details of MMR function are emerging via a convergence of biochemical and structural studies, genetic experiments, toxicology and cell biology analyses and, last but not least, clinical and pathological findings.

\section{MMR 101: the E. coli paradigm}

The biochemistry of MMR is well defined in Escherichia coli and the entire repair reaction has been reconstituted in vitro. Eleven biochemical activities function in three steps: initiation, excision and resynthesis. ${ }^{2,9}$ In the first step, the mismatch is detected by a homodimer of MutS, and upon interaction with a homodimer of MutL in the presence of ATP the single-strand endonuclease MutH is activated. MutH incises the newly synthesized DNA strand, which by definition contains the DNA synthesis error, at the closest GATC sequence located up to 1-2 kb away, either $5^{\prime}$ or $3^{\prime}$ to the mismatch. In E. coli, DNA methylation is used as the strand discrimination signal: MutH identifies the new strand for its transient lack of adenine methylation at the GATC site. For these features, this pathway of MMR is defined as methyl-directed, post-replicative or longpatch MMR. During the excision step, in the presence of DNA helicase II (MutU), single-strand exonucleases Exo I $\left(5^{\prime}>3^{\prime}\right)$, Exo VII, Exo X and RecJ (all $3^{\prime}>5^{\prime}$ ) remove the DNA tract between the incised, hemimethylated GATC site and the mismatch. Resynthesis is mediated by DNA polymerase III holoenzyme, single-strand binding protein, and finally, DNA ligase seals the nick. ${ }^{2,9}$

\section{Functional networks in DNA damage recognition: the advantage of combinatorial interactions}

Eukaryotic MMR has general features resembling those of the E. coli paradigm, such as the ability to perform strand discrimination, bidirectional excision and employment of the replicative DNA polymerases. ${ }^{2,3}$ However, multiple players at any given step are employed and the mechanistic details of their concerted actions are not fully understood.
In humans, at least five $\underline{\text { mutS }}$ homologue $(\mathrm{MSH})$ genes exist: $M S H 2, M S H 3, M S H 6$, which play a role in MMR, and $\mathrm{MSH} 4$ and $\mathrm{MSH} 5$, which are involved in meiotic recombination (Table 1). Phylogenetic and phylogenomic analyses revealed that these genes have evolved from Msh1, a direct descendant of MutS involved in MMR of mitochondrial DNA, which exists in yeast but not in mammalian cells (Table 1). Similarly, four mutL homologues are known: MLH1 (mutL homologue 1), MLH3, PMS2 and PMS1 (the latter two named after their yeast homologues, originally identified in a screen for postmeiotic segregation genes) (Table 1). No homologue of mutH has been hitherto identified in eukaryotes, thus leaving open the issue of how strand discrimination is performed (see below).

Whereas the bacterial MutS and MutL proteins are homodimers, their eukaryotic homologues function as heterodimers. Thus, human $\mathrm{MSH} 2$ interacts with $\mathrm{MSH} 6$ (also known as G:T-binding protein, GTBP) to form the complex named hMutS $\alpha,{ }^{10}$ and with MSH3 to form the complex named hMutS $\beta .^{11}$ An additional heterodimer is formed by $\mathrm{MSH} 4$ and $\mathrm{MSH} 5 .^{12}$

Similarly, MutL homologues are organized in heterodimers: human MLH1 and PMS2 combine to form hMutL $\alpha,{ }^{13}$ whereas $\mathrm{MLH} 1$ and PMS1 constitute hMutL $\beta ;^{14}$ more recently, a complex formed by $\mathrm{MLH} 1$ and the newly identified MLH3 has been described. ${ }^{15}$

The various combinatorial complexes perform specialized functions: hMutS $\alpha$ complex is involved in the repair of base-base mismatches and 1-bp IDLs, whereas the hMutS $\beta$ complex repairs IDLs of more than 1-bp but less than 12-bp (Figure 1). Longer IDLs up to 216-bp are repaired by a pathway independent of MMR. ${ }^{16}$ However, based on the mutational spectrum of MMR-deficient cell lines and on gene reintroduction experiments, hMutS $\beta$ may also play an accessory role in the repair of basebase mismatches. ${ }^{17}$ The meiosis-specific $\mathrm{MSH} 4$ and MSH5 proteins (Figure 1) lack an aminoterminal domain essential for the recognition of mispaired/misaligned bases and therefore do not participate in the repair of mismatched bases and IDLs. ${ }^{18}$

Table 1 Eukaryotic homologs of bacterial mut genes

\begin{tabular}{|c|c|c|c|c|c|}
\hline \multirow[b]{2}{*}{ E. coli } & \multirow[b]{2}{*}{ S. cerevisiae } & \multirow[b]{2}{*}{ H. sapiens } & \multirow[b]{2}{*}{$\begin{array}{c}\text { Chromosome } \\
\text { location }\end{array}$} & \multicolumn{2}{|c|}{ Mutations in human cancer } \\
\hline & & & & $\begin{array}{l}\text { Hereditary } \\
\text { (germline) }\end{array}$ & $\begin{array}{l}\text { Sporadic } \\
\text { (somatic) }\end{array}$ \\
\hline \multirow{6}{*}{ mutS } & Msh2 & MSH2 & 2p22-p21 & $\checkmark$ & $\checkmark$ \\
\hline & Msh3 & $\mathrm{MSH} 3$ & $5 q 11-q 12$ & & $\checkmark$ \\
\hline & Msh6 & MSH6 & $2 p 16$ & $\checkmark$ & $\checkmark$ \\
\hline & Msh4 & $\mathrm{MSH} 4$ & $1 \mathrm{p} 31$ & & \\
\hline & Msh5 & MSH5 & $6 p 21.3$ & & \\
\hline & Msh1 & a & - & & \\
\hline \multirow[t]{4}{*}{ mutL } & Mlh1 & MLH1 & $3 p 21.3$ & $\checkmark$ & $\boldsymbol{S}^{\mathrm{b}}$ \\
\hline & Pms1 & PMS2 & $7 p 22$ & $\checkmark$ & $\checkmark$ \\
\hline & Mlh2 & PMS1 & $2 q 31-q 33$ & $\checkmark$ & \\
\hline & MIh3 & $M L H 3$ & $14 q 24.3$ & & $\checkmark$ \\
\hline mutH & a & a & - & & \\
\hline mutU (uvrD) & a & a & - & & \\
\hline
\end{tabular}

aNot identified. ' Usually, loss of expression by promoter hypermethylation 
A
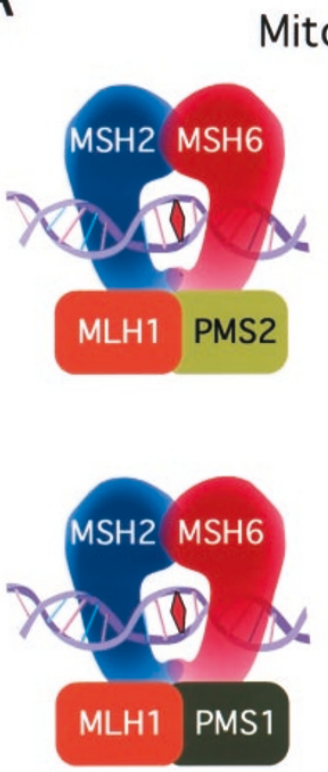

base-base mismatch 1-bp insertion/deletion loop

\section{Mitotic cell}
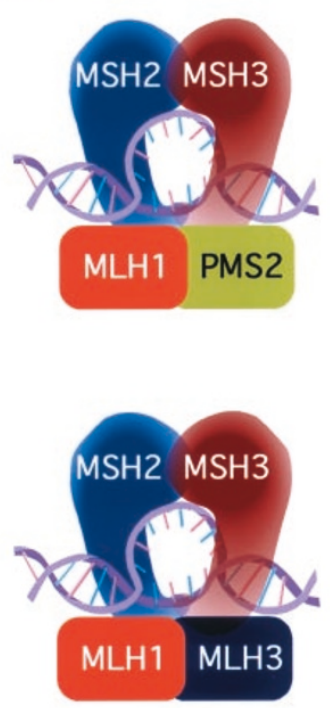

>1-bp insertion/deletion loop
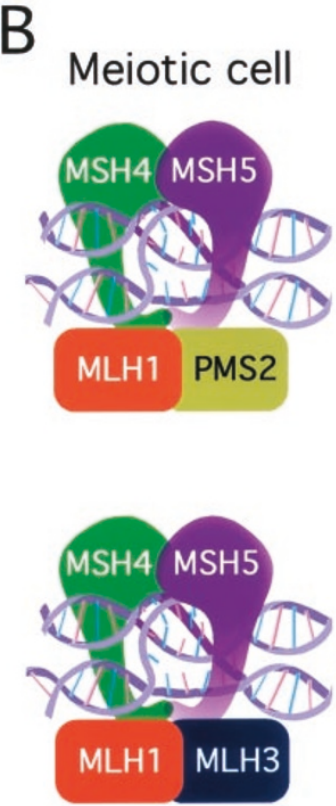

recombination intermediate

Figure 1 Functional networks in DNA damage recognition by MMR proteins. In mitotic and meiotic cells, MutS and MutL homologs form at least six multimeric complexes. In mitotic cells (A), these complexes recognize base-base mismatches (depicted as a red diamond) and insertion/deletion loops. In meiotic cells (B), the MSH4-MSH5-based complexes are likely involved in the recognition of recombination intermediates such as Holliday junctions. It should be noted that there is no biochemical proof of the direct interaction between MSH4-MSH5 and MLH1-PMS2 or MLH1-MLH3. See text for details

MutS and MutS homologues have ATPase activity, ${ }^{19,20}$ and more recently, based on structural similarities to HSP90 and DNA gyrase, MutL was also found to have ATPase activity $^{21}$ (see below). Accumulating evidence points to the existence both in yeast and mammalian cell extracts of higher order complexes of MutS and MutL homologues, ${ }^{22,23}$ however the precise functional properties of these interactions are not clearly defined. The hMutL $\alpha$ heterodimer associates with both hMutS $\alpha$ and hMutS $\beta$, participating in the repair of both mismatches and IDLs and providing most of the MutL-function in mutation avoidance ${ }^{24}$ (Figure 1). The other two MLH1containing complexes provide a minor role in mutation avoidance ${ }^{24}$ the functional properties of hMutL $\beta$ are poorly defined and the MLH1-MLH3 complex is likely to function in IDL repair; ${ }^{15}$ based on analogies with the yeast system, ${ }^{25}$ the MLH1-MLH3 complex may also play a role in meiotic recombination (Figure 1).

Thus, based on the available evidence obtained in mammalian systems and on extrapolations from the yeast studies, at least six complexes of MutS and MutL homologues exist in mitotic and meiotic cells (Figure 1).

\section{Structural insights into the function of MMR proteins}

In the past few years, structural biology successfully entered the MMR field, offering direct confirmation of previous biochemical observations and producing remarkable insights into the function of MMR proteins.
Recently, the crystal structure of $E$. coli Mut $S$ bound to a G:T mismatch and of Thermus aquaticus (Taq) MutS bound to DNA containing an unpaired $T$ were determined. ${ }^{18,26}$ The two structures are very similar: MutS functions as a dimer with the general shape of two 'opposing commas' or 'praying hands' joined by interactions at a single, composite ATP-binding site on one side, and at the bound mispaired/unpaired DNA on the opposite side, immediately suggesting allosteric communication between DNA binding and ATP binding and hydrolysis. The dimer encircles two channels, with diameters of approximately 30 and $40 \AA$, the latter being occupied by the mismatched DNA. ${ }^{18,26}$

Each subunit is formed by five flexible domains ensuring versatility in DNA damage recognition; however, upon mismatch binding, the structure is stabilized. Mismatch binding involves substantial conformational changes in each monomer and a sharp $\left(\sim 60^{\circ}\right)$ kinking of the mismatched DNA towards the major groove, widening and flattening the minor groove. ${ }^{18,26}$ This double induced fit of MutS and mismatched DNA may explain the remarkable mismatch detection properties of MutS proteins over a large background of normal DNA structures. $^{27}$ The five domains are defined as I, 'mismatch binding'; II, 'connector'; III, 'core'; IV, 'DNA clamp'; and V, 'ATPase'. Whereas the mismatch binding and DNA clamp domains are involved in binding to mismatched DNA, the connector and core domains constitute the backbone of each subunit and are 
involved in transmitting allosteric information of bound DNA co-factor to the ATPase domain. ${ }^{18,26}$

Interestingly, in both structures, the same FXE motif in domain I, is involved in binding to the G:T mismatch and to the unpaired $\mathrm{T}$; with the phenylalanine aromatic ring stacking onto the $\mathrm{T}$ and the glutamic acid carboxyl group hydrogen-bonding to the T. Remarkably, only one subunit interacts with the mismatched $\mathrm{T}$, creating an asymmetry in the dimer. In eukaryotes, the asymmetry is reflected by the heterodimers MSH2-MSH6 and MSH2-MSH3. This suggests that $\mathrm{MSH} 6$ and $\mathrm{MSH} 3$ are the subunits involved in mismatched/misaligned DNA recognition. By lacking a large segment of domain I, MSH4 and MSH5 are not involved in mismatch recognition: the absence of this domain would create a single $\sim 70 \AA$ diameter channel in the MSH4 and MSH5 dimer that could easily accommodate, side by side, two DNA molecules such as Holliday junctions during recombination. ${ }^{18}$

The ATPase domains are located on the opposite sides of the dimer, away from the bound DNA, and are interleaved with each other. They contain the Walker A and $B$ motifs, and their general fold resembles that of the ABC superfamily of ATPases, which includes transporters such as the cystic fibrosis protein, CFTR, and other DNA repair molecules, such as the double strand break repair enzyme, Rad 50. Like other ABC superfamily members, each ATPase active site is composite, i.e. shared between the two subunits across the dimer interface, an arrangement that is likely to coordinate conformational changes of both monomers. ${ }^{18,26,28}$

The MutS dimer is also asymmetric with respect to nucleotide binding. Thus, in $E$. coli MutS crystal, only the ATP binding site of the mismatch-binding subunit is occupied by $A D P ; 26$ and despite the presence of two ADP molecules in each domain V of Taq MutS, a structural asymmetry is evident. ${ }^{28}$ At the moment, crystals of the ATP-bound form of MutS are not available. Major conformational changes take place in MutS and MutS homologues after ADP/ATP binding and ATP hydrolysis. These changes have been detected by electron microscopy and partial proteolysis studies ${ }^{29}$ and are reflected by the cracking of MutS-DNA crystals after immersion in ATPcontaining solution. ${ }^{28}$ Based on similarities with the structure of the composite ATPase sites of Rad 50, available in the ATP-bound state, it has been proposed that a domain $\mathrm{V}$ helix is repositioned after $\gamma$-phosphate binding. The induced conformational change can then be transduced via the relatively rigid domain II/III (transmitter region) to the DNA binding domains, modulating the interaction with DNA. ${ }^{30}$

The crystal structure of the N-terminal region of MutL (named LN40), containing all the conserved residues, has been determined. ${ }^{21,31}$ Whereas the $\mathrm{C}$-terminal region of MutL mediates constitutive dimerization, the $\mathrm{N}$-terminal region undergoes ATP/ADP-regulated dimerization during MMR. In fact, LN40 is a partially unstructured, elbowshaped, two-domain monomer that resembles DNA gyrase and the chaperone HSP90. Upon binding to the nonhydrolyzable ATP analog, ADPnP, LN40 becomes well ordered, dimerizes and a large DNA binding groove is formed in the middle of the dimer. Dimerization is mediated mostly by the association of the first domains, each binding a nucleotide molecule. ${ }^{21,31}$ Given the sequence conservation, MutL homodimerization is likely to be reflected by heterodimerization of eukaryotic MutL homologues.

Upon ADPnP-induced dimerization, two potential interfaces for interaction with MutS and MutH are formed. In turn, dimerization leads to ATP hydrolysis, generating an ADP-bound dimer. The latter is more relaxed, decays to monomers, and eventually to the nucleotide-free form, which does not activate MutH. Thus, like MutS, MutL is also a molecular switch and is regulated by cycles of ATP binding and hydrolysis. ${ }^{31}$

Modeling data suggest that MutL signaling to MutH may be regulated by structural modifications. MutH structure has homology to type II restriction endonucleases, but, in keeping with its single strand endonuclease activity, it is a monomer. It has the shape of a two-domain open clamp delimiting a DNA binding cleft. The active site is located at the bottom of the cleft and unlike restriction enzymes is conditionally regulated. Regulation appears to be mediated by MutL conformational changes transmitted to the MutH Cterminal helix that acts as a lever, causing a rotation of the two domains and orienting them for catalysis. Thus, the general theme of conformational switches is further exploited in $\mathrm{MutH}^{32}$

\section{Signaling complexes in DNA repair: three models for one problem, strand discrimination}

A central problem in MMR concerns the recognition of the mismatch or other DNA damage and the assembling of a multimeric complex able to coordinate subsequent repair events, including strand discrimination, and possibly transduction of additional responses, such as cell cycle arrest and apoptosis. The adenosine nucleotide binding property and ATPase activity of MutS and MutL proteins point to their signaling features.

It was originally hypothesized that MSH2-MSH6 might act as a molecular switch regulated by a cycle of ADP/ATP binding and ATP hydrolysis to ADP, much as the G protein family members are regulated by guanosine nucleotides during transmembrane signal transduction. ${ }^{33,34}$ In this model, MSH2-MSH6 is capable of high-affinity mismatch recognition in its ADP-bound state and mismatch binding acts as nucleotide exchange factor provoking the ADP-ATP exchange. The ATP-bound form of MSH2-MSH6 undergoes a conformational change into a clamp with reduced affinity for the mismatch and diffuses freely along the DNA in an ATP hydrolysis-independent fashion ('sliding clamp'), signaling to additional components of the MMR machinery (and above a threshold of DNA damage to pro-apoptotic mediators) (Figure 2A). ${ }^{29,35}$ Signaling to the replicative polymerase may change the polymerase's conformation in such a way that the forward processivity is stopped and the $3^{\prime}>5^{\prime}$ exonuclease activity is activated, removing the newly synthesized DNA strand. ${ }^{29,35}$ It should be noted that these latter aspects of the sliding clamp model are at the moment hypothetical; however, this model explains well the directionality of the excision towards the mismatch (and 


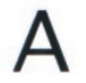

\section{Sliding clamp}

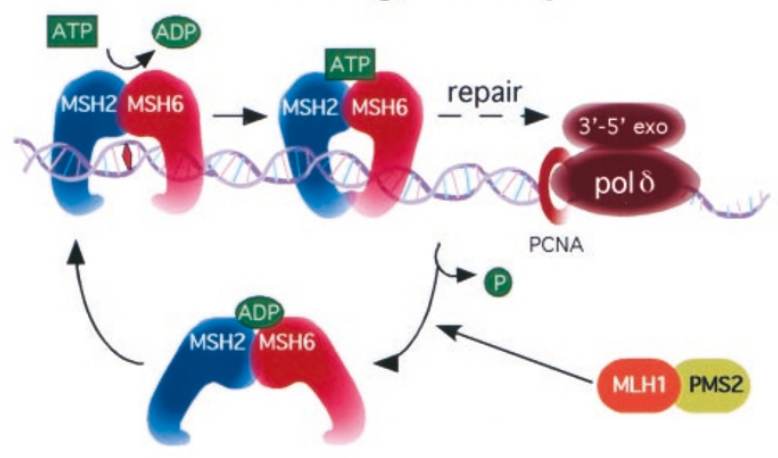

B

\section{ATP-dependent translocation}
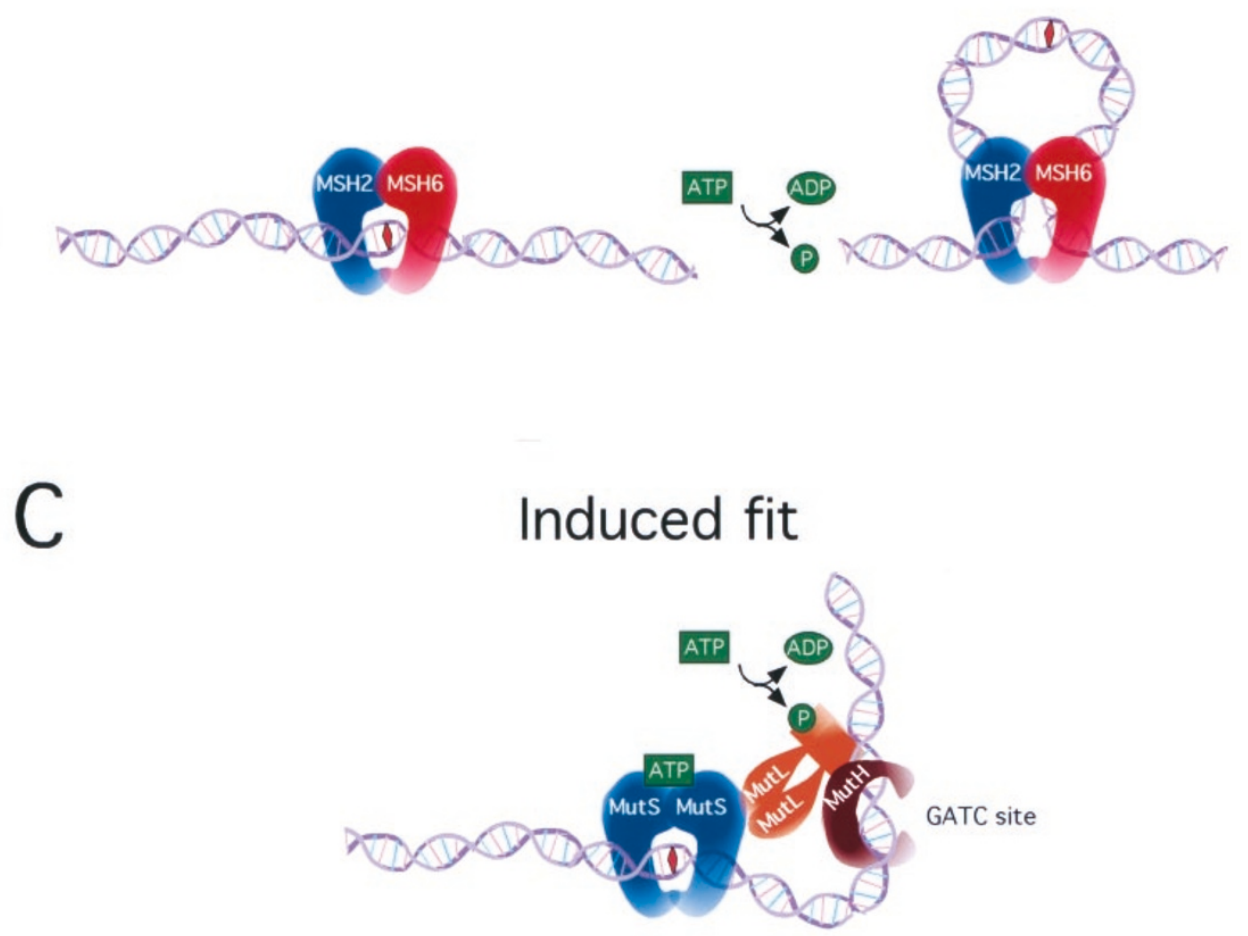

Figure 2 Models of the signaling properties of MMR proteins in mismatch recognition and subsequent repair events. (A) Mismatch binding acts as an ADP-ATP exchange factor; the ATP bound MSH2-MSH6 complex becomes a 'sliding clamp' that diffuses freely in an ATP-hydrolysis-independent fashion along the DNA, signaling to additional components of the MMR machinery and to the replicative DNA polymerase. Upon ATP hydrolysis that is perhaps stimulated by MLH1-PMS2, the MSH2-MSH6 complex is released from DNA. (B) In the 'ATP-dependent translocation' model, hMutS $\alpha$ upon mismatch binding moves away from the mismatch along the helix contour by using the energy of ATP hydrolysis, thus linking mismatch recognition to the strand break or DNA terminus that directs excision to the newly synthesized strand. (C) The 'induced fit' model precludes dissociation of MutS from the mismatch. Subsequent repair events, including strand discrimination, are coordinated via direct protein-protein interactions with other MMR proteins, such as MutL and MutH. In this model, ATP is required as a 'proofreading' means to verify mismatch recognition by MutS and allow subsequent signaling events. Signaling to MutH requires ATP-hydrolysis by MutL. Additional details of the three models are presented in the text

resynthesis in the opposite direction). In this model, tethering of the MMR machinery to the replicative polymerase would allow strand discrimination. The direct interaction of $\mathrm{MLH} 1, \mathrm{MSH} 3$, and $\mathrm{MSH} 6$ with the polymerase processivity factor PCNA (see below) may link MMR to DNA replication: the DNA termini of the leading strand would provide the strand discrimination signal by marking the newly synthesized DNA. ${ }^{2,3}$

A second model ("ATP-dependent translocation") (Figure $2 \mathrm{~B}$ ) is based initially on electron microscopic visualization of $E$. coli MutS bound to heteroduplex DNA: ${ }^{20}$ MutS extrudes a DNA loop in an ATP hydrolysis-dependent 
manner generating $\alpha$ - or $\Omega$-shaped structures with the mismatch located within the loop. ${ }^{3,20}$ In this model, recognition of mismatched DNA by hMutS $\alpha$ does not require ADP binding, although the presence of ADP increases mismatch binding specificity. ${ }^{36}$ ATP binding then stimulates dissociation of hMutS $\alpha$ from the mismatch and ATP hydrolysis provides the energy for translocation of hMut $\alpha$ along the helix contour. ${ }^{36}$

In both models, hMutS $\alpha$ leaves the mismatch, diffusing along the DNA, and can signal subsequent repair events, thus linking mismatch recognition to the strand break or DNA terminus that directs excision to the newly synthesized strand. $^{35,36}$ Very recently, a third model has been proposed on the basis of the crystal structure of bacterial MutS proteins bound to mismatches, which challenges the idea that MutS proteins leave the mismatch. ${ }^{18,26}$ Based on the sharp kinking of mismatched DNA and the conformational changes of MutS upon DNA binding, in this 'induced fit' model it is argued that MutS does not leave the mismatch and marks subsequent repair events, including strand discrimination, via direct protein-protein interactions with other MMR proteins, such as MutL and MutH ${ }^{18,26}$ (Figure 2C). A critical observation in support of this model is that, in the presence of MutL and ATP, MutS can activate MutH-mediated cleavage in trans, i.e. the mismatch and the hemimethylated GATC site can reside on two separate DNA molecules. ${ }^{28}$ In this model, the requirement of ATP is explained as a 'proofreading' means to verify mismatch recognition by MutS, as ATP binding reduces the MutS affinity for homoduplex DNA more than for heteroduplex DNA. Furthermore, ATP would play a second role, of authorization of subsequent repair events, as only ATP binding by MutS would allow productive interaction with MutL. 28

Clearly, additional biochemical, biophysical and structural studies are necessary to establish the relative merits of these models and dissect the discrete temporal and spatial steps involved in MMR initiation. Certainly, the structural biology data discussed earlier support the notion of MutS conformational switch(es) induced by cycles of nucleotide binding and hydrolysis. ${ }^{29}$ However, in the presence of ATP and MutL (or MutL homologues), MutS (or MutS homologues) do(es) not seem to leave the mismatch. ${ }^{37-39}$ The extrusion of the $\alpha$ - or $\Omega$ shaped loop visualized by electron microscopy might be compatible with MutS not leaving the mismatch and threading the DNA through the second channel. A related explanation, more consistent with the crystallographic dimensions of the MutS dimer, is that electron microscopy visualized a MutS tetramer, with one dimer bound to the mismatch and the other threading the DNA. ${ }^{40,41}$ An advantage of models in which MutS does not leave the mismatch is that it can mark the site where exonucleolytic degradation can stop and DNA synthesis can resume.

How does MutS modulate MutL and MutH function? Via structural modeling based on crystallographic data, it has been proposed that a MutL dimer interacts with MutS transmitter region: through this region, the conformational changes of concomitant ATP and mismatch DNA binding can be signaled to MutL. ${ }^{18}$ In turn, MutL can signal to MutH via structural changes that depend on ATP hydrolysis. ${ }^{28}$ It is likely that coordinate cycles of nucleotide binding and hydrolysis in MutS and MutL regulate both MMR initiation and later events of DNA resynthesis. ${ }^{30}$ Given the high degree of sequence conservation, similar mechanisms relying on intra- and intermolecular allosteric communication are likely to take place in mammalian cells where they are further complicated by additional interactions such as those involving PCNA.

\section{Multiple roles of PCNA interactions in MMR}

Proliferating cell nuclear antigen (PCNA) is an auxiliary factor for DNA polymerase $\delta$ and $\varepsilon$ and is necessary for DNA replication. It is as a toroidal (doughnut-shaped) heterodimer that is loaded onto DNA by replicative factor C (RFC) in an ATP-dependent process, and acts as a sliding clamp that encircles template DNA and provides processivity to the associated DNA polymerase. PCNA is emerging as a key player linking early and late events during MMR and possibly mediating strand discrimination. PCNA entry in the MMR field was marked by its identification as an interactor of human MLH1 in a yeast two-hybrid screening. ${ }^{42}$ In addition, some non-lethal mutations of the pol30 gene (encoding the yeast PCNA) give MSI and a mutator phenotype, and are epistatic with MMR gene mutations. ${ }^{42,43}$

More recently, PCNA was found to interact with $\mathrm{MSH}$ and MSH6. ${ }^{44-46}$ The interaction occurs between the interdomain connector loop of PCNA and the conserved motif QXXL/IXXFF, located at the $\mathrm{N}$-terminus of $\mathrm{MSH} 3$ and MSH6 and also present in other PCNA-interacting proteins,

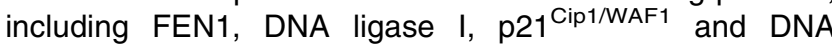
methyltransferase. Mutations of this conserved motif in yeast Msh3 and Msh6 abolish interaction with PCNA and yield a mutator phenotype. ${ }^{44-46}$

Incubation of MMR-proficient extracts with peptides corresponding to the PCNA interacting motif of p21 ${ }^{\text {Cip1/WAF1 }}, \mathrm{MSH} 3$ and MSH6 inhibits MMR at the initiation step, i.e., before excision and DNA resynthesis. ${ }^{42,44,46}$ Co-immunoprecipitation experiments, demonstrating an interaction with MSH2, MHL1 and PMS2, also place PCNA at the initiation step. ${ }^{23}$ During MMR initiation, PCNA may have a double role. It increases specificity of mismatch recognition by Msh2-Msh6. ${ }^{45}$ However, PCNA also disrupts ternary complexes of Msh/Mlh heterodimers and mismatched DNA upon loading onto DNA, and causes the Msh/Mlh complex to slide away from the mismatch. ${ }^{47}$ Thus, after an early role in facilitating mismatched DNA binding, PCNA may subsequently help mobilizing the $\mathrm{MSH} /$ MLH complex away from the mismatch in search of the strand discrimination signal. ${ }^{47}$ Strand discrimination might be provided minimally by the interaction of MMR proteins with the replicative polymerase via PCNA: in this way, DNA termini at the replication fork would be readily accessible to MMR proteins. A direct association of MMR proteins with DNA replication is confirmed by the colocalization of $\mathrm{MSH} 3$ and MSH6 with PCNA and newly replicated DNA. ${ }^{46}$

Finally, after excision, PCNA would participate in the DNA resynthesis step. ${ }^{23}$ Since the PCNA homotrimer cannot accommodate all the PCNA interactors, its interactions need to be transient and dynamically change as the MMR reaction progresses. 


\section{Additional interactions in MMR during excision and resynthesis}

After MMR initiation, additional protein-protein interactions mediate subsequent repair steps. In E. coli, the excision step is mediated by $5^{\prime}>3^{\prime}$ and $3^{\prime}>5^{\prime}$ exonucleases but requires MutS and MutL. Not surprisingly, a direct interaction between the human $5^{\prime}>3^{\prime}$ exonuclease EXOI/HEXI and $\mathrm{MSH} 2$ has been detected. ${ }^{48}$ Based on the mutator phenotype of yeast Exo I mutants, EXOI/HEXI is likely to play a role in MMR. This is confirmed by the detection of germline mutations of the EXO1 gene ${ }^{49}$ in patients affected with hereditary colorectal cancer, the disease linked to MMR gene mutations (see below).

Human polymerase $\delta$ and perhaps $\varepsilon$ effect DNA resynthesis. ${ }^{50}$ This process might be regulated by the above-mentioned interaction between MMR proteins and PCNA and also involves the single-strand binding, heterotrimeric complex RPA. Finally, the ligase involved in human MMR is likely to be DNA ligase I, based on its association with DNA polymerase $\delta$ and PCNA. ${ }^{3}$

The multiple interactions occurring during MMR are depicted in Figure 3.

\section{Mutations of MMR genes in cancer: disease causation by faulty interactions}

Mutations in MMR genes determine a status of genomic instability that is associated with predisposition to cancer (Table 1). Many reviews have comprehensively covered the relationship between MMR gene defects and cancer. ${ }^{51}$
Germline mutations of MMR genes are found in families affected with hereditary nonpolyposis colorectal cancer (HNPCC) or Lynch syndrome, an autosomal dominant disease predisposing to cancer of the colon, endometrium, stomach, ovary, urinary and biliary tracts. ${ }^{51}$ Somatic mutations of MMR genes and epigenetic inactivation of $\mathrm{MLH} 1$ are also found in $12-15 \%$ of sporadic colorectal cancer cases and in a variable fraction of cancers of the endometrium, stomach and other sites. Due to unrepaired slippage intermediates originated during replication of simple repetitive sequences, tumors from HNPCC individuals and sporadic MMR-defective tumors manifest microsatellite instability (MSI). ${ }^{52,53}$

Pathogenetic mutations of MMR genes often involve frameshift-inducing insertions/deletions, non-sense mutations, or changes in critical residues directly affecting catalytic (ATP binding/hydrolysis) or DNA binding activity. However, in some cases, disease-causing missense mutations are pathogenetic because they disrupt critical proteinprotein interactions during MMR. Thus, several MLH1 missense mutations found in HNPCC kindreds cluster in the $\mathrm{C}$-terminal region of the protein and impair the interaction with PMS2. ${ }^{54}$ Interestingly, similar studies concluded that several HNPCC mutations in $\mathrm{MSH} 2$ do not affect its interaction with $\mathrm{MSH}^{55}$ Finally, based on structural considerations modeled on bacterial MutS proteins, several $\mathrm{MSH} 2$ mutations in the transmitter region linking the ATPand DNA-binding sites may impair the interaction and signaling with $\mathrm{MLH} 1{ }^{18,26}$ Thus, aberrations in both intraand intermolecular interfaces of the MMR heterodimers hMutL $\alpha$ and hMutS $\alpha$ play a direct role in cancer causation.

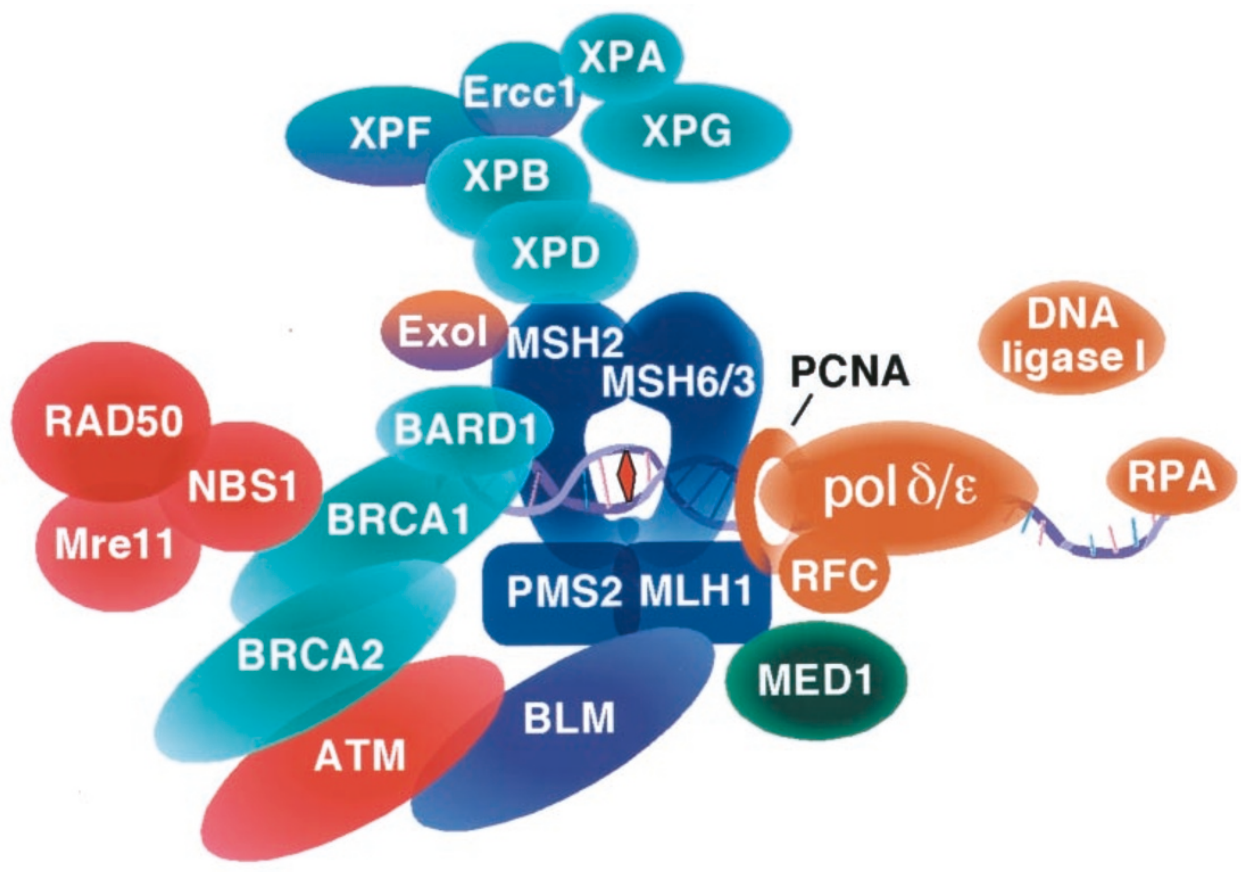

Figure 3 Schematic of multiple interactions of MMR proteins. Core MMR proteins (in blue) interact with multiple factors, in reactions that are relevant to MMR (orange), BER (green), TCR (aqua), recombination (purple), apoptosis/cell cycle checkpoint signaling (red). Factors participating in multiple reactions, such as Exol, XPF and Ercc1 are appropriately shaded. It should be noted that the interactions between MSH2 and NER/TCR factors have been demonstrated in yeast, whereas the human NER/TCR homologues are depicted here. See text for details 


\section{Beyond DNA mismatch correction: links to other DNA transactions}

In addition to a direct role in MMR, mammalian MMR proteins are involved in several DNA transactions, including transcription-coupled repair (TCR), base excision repair (BER) and recombination and meiosis. Even though some extra-MMR functions of MMR proteins, such as their role in TCR and recombination, were initially recognized in bacteria, most studies of this emerging theme in MMR analysis are being conducted in yeast and mammalian cells.

\section{Role of MMR components in transcription-coupled repair}

Nucleotide excision repair (NER) is a versatile DNA repair system involved in the removal of a variety of lesions ranging from UV light-induced pyrimidine dimers to bulky adducts, such as those generated by benzo[a]pyrene and cisplatin. ${ }^{56,57}$ Although structurally unrelated, these lesions share the ability to distort the helix and covalently modify the DNA. ${ }^{56}$ Approximately 30 polypeptides are involved in NER at the steps of DNA damage recognition, excision of the lesion, resynthesis, and ligation. ${ }^{56,57}$ Two NER pathways are known: global genome repair (GGR), a general pathway involved in unbiased repair throughout the genome, and transcription-coupled repair (TCR), a repair system that preferentially corrects lesions in the transcription template strand of RNA polymerase II-transcribed genes. ${ }^{58,59}$

Studies in E. coli, yeast, and mammalian cells demonstrate the involvement of MMR proteins in TCR. Both mutS and mutL, but not mutH, E. coli mutants show reduced TCR of UV damage and slightly increased UV sensitivity. Similarly, human cancer cell lines and lymphoblastoid cultures harboring defects in MSH2, MLH1 and PMS2 do not exhibit TCR of UV damage, i.e. there is no difference in repair of the transcribed versus the non-transcribed strand. These cancer cell lines also show increased sensitivity to UV irradiation. ${ }^{60}$ These findings have been somehow mitigated by subsequent studies, which failed to detect UV sensitivity of human MMR-deficient cancer cell lines and showed reduction, but not abrogation, of UV damage TCR in MSH2- and MLH1-deficient cell lines. ${ }^{61}$ The discrepancy might be due to the different methods used to measure repair on the transcribed versus the nontranscribed strands and might reflect the absolute requirement of MSH2 and MLH1 in TCR of only some specific UVinduced lesions, such as pyrimidine dimers, but not (6-4) photoproducts. ${ }^{61}$

TCR can also occur outside of the NER realm, i.e., in the repair of oxidative damage induced by ionizing radiation, which may proceed via the action of DNA $N$-glycosylases in a pathway of base excision repair. Interestingly, $\mathrm{MSH} 2$, but not MLH1, is involved in TCR of oxidative damage; TCR of specific oxidative lesions, thymine glycols induced by $\mathrm{H}_{2} \mathrm{O}_{2}$, is completely absent in MSH2-defective cell lines, ${ }^{61}$ and TCR of 8-oxoguanine appears to be reduced in human tumor cell lines devoid of MSH2. ${ }^{62}$ Yeast mutants for the Msh2, MIh1, and Pms1 genes are deficient in TCR of thymine glycols, but, unlike mammalian cells, do not show alterations in TCR of UV repair.

Although the mechanistic details of the involvement of MMR proteins in TCR are not known, specific interactions between Msh2 and NER proteins have been detected in yeast. ${ }^{63}$ In S. cerevisiae, Msh2 was found to interact with Rad1, Rad2, Rad3, Rad10, Rad14 and Rad 25 (homologues of human XPF, XPG, XPD, ERCC1, XPA and XPB, respectively) (Figure 3). Since all these NER factors are required for TCR, their interaction with $\mathrm{MSH} 2$ may mediate the role of MMR proteins in TCR. ${ }^{63}$ One possibility is that via the interaction with Rad 3 and Rad 25 (human XPD and $\mathrm{XPB}$ ), which are also components of the basal transcription factor TFIIH, Msh2 contributes to the recognition of a stalled RNA polymerase on the transcription template strand at sites of DNA damage.

Additional mechanistic possibilities are that MMR proteins might function in TCR by sensing the DNA damage itself as the MSH2-MSH6 heterodimer can bind to some lesions repaired by NER via GGR or TCR, including cisplatin adducts ${ }^{64}$ and 8-oxoguanine: $A$ and 8oxoguanine:C mismatches. In fact, it has been suggested that binding of MMR proteins to 8-oxoguanine may in itself cause stalling of RNA polymerase, providing the initializing signal for TCR. ${ }^{62}$ Finally, MMR proteins may function later during resynthesis of the transcribed strand. ${ }^{61}$ It will have to be determined whether MMR and NER proteins are part of a single, multifunctional complex or whether distinct complexes exist that contain various complements of MMR and NER polypeptides. ${ }^{63}$

Regardless of the specific mechanistic role, the involvement of MMR proteins in TCR of UV and oxidative damage suggests that inactivation of MMR function may lead to the accumulation of both environmental and endogenous oxidative DNA lesions, including those generated during normal metabolism. This might contribute to the pathogenesis of hereditary and sporadic colorectal cancer.

\section{MMR and TCR: a link to BRCA1 and BRCA2?}

The tumor suppressor genes BRCA1 and BRCA2 are responsible for the majority of the cases of hereditary predisposition to breast and ovarian cancer. In addition to their role in the repair of DNA double-strand breaks, BRCA1 and BRCA2 are also involved in TCR. Mouse embryonic stem (ES) cells with homozygous inactivation of Brca1 and human cancer cells containing mutated BRCA1 or $B R C A 2$ genes display reduced TCR of the oxidative damage, including thymine glycols and 8-oxoguanine, generated by exposure to ionizing radiation and hydrogen peroxide, but the global genome repair (GGR) of oxidative damage is not affected. ${ }^{62,65,66}$ Interestingly, TCR of UV damage is normal in murine $B r c a 1^{-1-}$ ES cells, suggesting a specific requirement of BRCA1 in TCR of oxidative damage. ${ }^{65}$ This is reminiscent of the opposite obligatory role of MLH1 in TCR of UV, but not oxidative damage. ${ }^{61}$ Consistently, Brca1-1- ES cells and BRCA1-defective breast cancer cells are hypersensitive to ionizing radiation and hydrogen peroxide, but not UV irradiation. ${ }^{65,66}$ This 
hypersensitivity and the defective TCR of oxidative damage can be corrected by re-expression of BRCA1. ${ }^{62,66}$

These observations raise the possibility that MMR and BRCA proteins work coordinately in TCR of oxidative damage. This possibility has been strengthened by the recent finding that a large ( $>2$ MDaltons) complex of BRCA1-associated proteins, named BASC (for BRCA1associated genome surveillance complex), contains $\mathrm{MSH} 2$, MSH6, MLH1 and, by inference, PMS2, in addition to ATM, BLM and RAD50-MRE11-NBS1. ${ }^{67}$ It is also known that BRCA1 interacts and colocalizes with BRCA2 (Figure 3). It is possible that the MMR proteins, in particular the heterodimer $\mathrm{MSH} 2-\mathrm{MSH}$, provide a DNA damage recognition signal that is required for the BRCA1- and BRCA2dependent TCR. ${ }^{59,67}$ Alternatively, via its interaction with RNA polymerase II and basal transcription factors TFIIH, TFIIE and TFIIF, BRCA1 may facilitate identification of the transcribed strand by MMR proteins or coordinate active transcription with repair of the transcribed strand. ${ }^{67}$

BRCA1 interacts directly with $\mathrm{MSH} 2, \mathrm{MSH} 3$ and $\mathrm{MSH} 6$, and the BRCA1 interactor BARD1 associates with MSH2. ${ }^{68}$ Interestingly, BRCA1 appears to display high affinity for the ATP-bound but not ADP-bound form of the MSH2-MSH6 heterodimer, suggesting that BRCA1 may be involved in regulated signaling to downstream events during repair of oxidative damage/mismatched bases. ${ }^{68}$

Although the binding interfaces and the mechanistic details of the interaction between MMR and BRCA proteins require additional investigation, it is certainly interesting that mutations in the respective genes are associated with cancer predisposition. Intriguingly, although some specific Ashkenazim mutations in BRCA1 and BRCA2 are not linked to an excess of colorectal cancer, it has been reported that $B R C A 1$ - and BRCA2-mutation carriers are at increased risk of developing colorectal and other gastrointestinal cancers. Similarly, it has been proposed that breast cancer be considered an integral component of the tumor spectrum of HNPCC. Its incidence seems to differ in HNPCC families that are $\mathrm{MSH} 2$ mutation-positive (breast cancer under-represented) versus those that are $\mathrm{MLH} 1$ mutation-positive or mutation-negative (breast cancer over-represented). Certainly, delineation of the target organ profile of defective MMR or $B R C A$ genes will stem from a more complete understanding of their functional interactions in DNA repair and related processes.

\section{Interactions of MMR components with base excision repair: MED1 and the possible role of DNA methylation in DNA repair}

The recent identification of the DNA $N$-glycosylase MED1 as an interactor of $\mathrm{MLH} 1$ provides an interesting link between MMR and BER, and reveals initial clues to an unprecedented role of DNA methylation in DNA repair processes in humans. 69

The repair patches of NER and MMR systems comprise 24-34 bases- and 1.5-2 kilobases-long oligonucleotides, respectively. On the contrary, BER usually acts in a lesionspecific manner on individual DNA bases: DNA $N$-glycosylases, the initial enzymes in BER, remove damaged bases via the cleavage of the $N$-glycosylic bond. ${ }^{70-73}$ The ensuing apurinic/apyrimidinic (AP) site is then converted into a singlenucleotide gap by AP endonucleases and $3^{\prime}$ - and $5^{\prime}$ phosphodiesterases. DNA polymerase $\beta$ and DNA ligase complete the repair by filling-in and sealing the gap. ${ }^{70,71}$

BER is mostly involved in the repair of endogenous oxidative lesions of bases caused by reactive oxygen species generated during aerobic metabolism, and of exogenous lesions, such as alkylation of purines and formation of etheno adducts, caused by alkylating agents and the carcinogens vinyl chloride and ethyl carbamate. $^{70-74}$

However, in some special instances, BER acts on mismatches via the action of mismatch-specific DNA $N$ glycosylases. Thus $E$. coli MutY and its human homologue MYH act as adenine glycosylases on $A: C$ and $A: G$ mismatches as well as on adenine paired with the oxidative lesion, 8-oxoguanine. ${ }^{70,71}$ Recently, a second activity distinct from MMR and operative on $A: C$ mismatches has been described. ${ }^{75}$ Finally, mismatch-specific uracil and thymine glycosylases, such as TDG, are known to act on $\mathrm{G}: U$ and $\mathrm{G}: T$ mismatches generated by spontaneous hydrolytic deamination of cytosine and 5-methylcytosine to uracil and thymine, respectively. ${ }^{76}$

These mismatch-specific activities of BER imply coordination with MMR in order to minimize the possibility of inducing mutations during repair. Thus, for a G:A mismatch due to misincorporation of the $G$ on the newly synthesized strand, MMR would correctly repair to $T: A$, but removal of the adenine from the old strand by MYH would cause a G:C transversion mutation. The molecular details of this likely coordination between MMR and BER are not known. However, the recent cloning of the DNA repair enzyme MED1 may lead to a clarification of this problem. ${ }^{77}$

The human MED1 protein was identified as a MLH1 interactor in a yeast two-hybrid screening ${ }^{69}$ (Figure 3). MED1 protein contains an $\mathrm{N}$-terminal 5-methylcytosine binding domain (MBD) and a C-terminal catalytic region with homology to bacterial BER DNA N-glycosylases/ lyases, such as E. coli endonuclease III and MutY, Methanobacterium thermoautotrophicum Mig.Mth, and Micrococcus luteus UV-repair endonuclease. ${ }^{69}$ This structure implies a novel, unprecedented role of DNA methylation in eukaryotic DNA repair processes, and is reminiscent of the role of adenine methylation in bacterial MMR. ${ }^{69}$ MED1 binds to oligonucleotides containing 5methylcytosine on one or both strands (hemimethylated or fully methylated DNA), and has endonuclease activity on a supercoiled plasmid DNA substrate, hence the name MED1 (methyl-CpG-binding endonuclease 1). ${ }^{69}$

Originally, it had been proposed that MED1 might function in long-patch MMR as a candidate eukaryotic homologue of $E$. coli $\mathrm{MutH}$, in analogy to the MutL-MutH interaction in this system; or that it may work in a shortpatch MMR pathway, similar to the E. coli Vsr endonuclease, which also interacts with MutL. ${ }^{69}$ However, MED1 does not seem to display endonuclease activity on double-stranded oligonucleotides, ${ }^{78}$ and subsequent experiments appear to rule out a possible role of MED1 and DNA methylation in MMR strand-discrimination in vitro. ${ }^{79}$ 
More recently, MED1, also known as MBD4, was shown to act as a $\mathrm{G}: T$ and $\mathrm{G}: \mathrm{U}$ mismatch-specific thymine and uracil glycosylase. ${ }^{78,80}$ The glycosylase activity of MED1 preferentially targets $\mathrm{G}: T$ and $\mathrm{G}: \mathrm{U}$ mismatches in the context of methylated and unmethylated CpG sites. ${ }^{78,80,81}$ This suggests that MED1, like its cognate human mismatch-specific thymine and uracil glycosylase TDG, ${ }^{76}$ is involved in the repair of deaminated 5-methylcytosine and cytosine at CpG sites. ${ }^{77}$ Although MED1 and TDG do not display any sequence homology, their similar biochemical activity raises the issue of functional redundancy.

Mutagenesis by deamination of 5-methylcytosine to thymine at $\mathrm{CpG}$ sites is a prominent force of genetic instability in the evolution of primates, in human evolution and genetic variation, and in cellular evolution during tumorigenesis (references $\mathrm{in}^{77}$ ). Indeed, it is estimated that a large fraction of cancer mutations, including nearly $50 \%$ of somatic mutations of the tumor suppressor gene TP53 in colorectal and brain cancer are $\mathrm{G}: \mathrm{C}$ to $\mathrm{A}: \mathrm{T}$ transitions at CpG sites, the result of unrepaired deamination events. Since G:T and G:U repair systems are generally effective in protecting cells from spontaneous mutagenesis, ${ }^{82}$ it is reasonable to hypothesize that inactivating mutations of TDG and MED1 may accelerate the accumulations of these transition mutations at CpG sites in TP53 and other cancer genes. Whereas TDG mutations in human cancer have eluded detection, the MED1 (MBD4) gene is mutated in human $\mathrm{MSI}$ colorectal, endometrial, pancreatic, and gastric carcinomas that exhibit a defect in the MMR genes $\mathrm{MLH1}$ and MSH2 (Yokozaki, personal communication). ${ }^{83,84}$ As a consequence of the generalized $\mathrm{MSI}$ due to $\mathrm{MLH1}$ or MSH2 inactivation, polyadenine microsatellites in the coding region of the MED1 gene contract or expand, causing frameshifts, which predict the synthesis of truncated proteins lacking the C-terminal catalytic domain. ${ }^{77}$ It should be pointed out that these are the only truncating mutations affecting a protein with DNA $\mathrm{N}$ glycosylase activity ever described in human cancer. In addition, in colorectal cancer loss of heterozygosity at the MED1 (MBD4) locus has been reported, ${ }^{84}$ lending support to the idea that this gene may act as a tumor suppressor of the caretaker type. Thus, a fraction of MSI human carcinomas are defective not only in long-patch MMR but also in MED1 thymine and uracil glycosylase activity. It remains to be demonstrated whether MED1 mutations are present in the more common microsatellite-stable (MSS) carcinomas.

Despite their potential antimutagenic function, the involvement of MED1 and TDG in tumorigenesis and their impact on mutation rate at $\mathrm{CpG}$ sites await formal demonstration. A related issue pertains to the possible redundancy of these two enzymes. Fundamental differences in the biochemical properties of TDG and MED1 have been uncovered. TDG preferentially targets $G: U$ and G:ethenocytosine mismatches over G:T mispairs. ${ }^{85}$ To the contrary, the $k_{\text {cat }}$ of MED1 for G:U and G:T mismatches is similar whereas the activity on G:ethenocytosine is very weak. $^{78,81}$ This suggests that MED1 may be the main guardian of genetic fidelity at $\mathrm{CpG}$ sites. In principle, in addition to these biochemical differences, the redundancy of MED1 and TDG might be limited by spatially/temporally restricted expression patterns and by functional differences pertaining, for instance, to the role of DNA methylation and the MBD, that is present in MED1 but not in TDG (see below).

What is the role of the MLH1-MED1 interaction? MED1 and MLH1 coimmunoprecipitate in human cells and the interaction appears to have functional consequences. Thus, transfection in MSS cell lines of a deletion mutant lacking the MBD domain but maintaining the region of interaction with MLH1 is associated with microsatellite instability of a $\beta$-galactosidase reporter gene. ${ }^{69}$ However, since this result was obtained with a MED1 deletion mutant and not with a null mutant, care should be used in the interpretation of the physiological significance of this experiment. Thus, the association with $\mathrm{MSI}$, rather than reflecting a direct role of MED1 in long-patch MMR, is more likely to be due to the MED1 deletion mutant sequestering MLH1 in mislocalized, non-functional complexes.

MED1 binds very tightly to the AP site reaction product generated by its glycosylase activity. ${ }^{78}$ This feature, also described also for TDG and MutY, may allow protection of the AP-site before processing by $\mathrm{AP}$ endonuclease, but may also signal a DNA damage checkpoint. ${ }^{78}$ Perhaps the interaction with $\mathrm{MLH} 1$ may serve a signaling role. $^{78}$ Alternatively, the interaction with MLH1 may allow a cross talk between long-patch MMR and BER in the repair of potentially conflicting lesions, such as G:T mismatches in the context of $\mathrm{CpG}$ sites due to the misincorporation of $\mathrm{G}$ in the newly synthesized strand opposite a $T$ (as discussed above for $\mathrm{MYH}$ and $\mathrm{G}: \mathrm{A}$ mismatches). Albeit misincorporation of $G$ opposite $T$ is rare, its repair by long-patch MMR would be antimutagenic, whereas processing by MED1 would lead to removal of the $T$ resulting in $A: T$ to $G: C$ transitions. ${ }^{77} \mathrm{~A}$ similar coordination has been proposed to occur in E. coli between long-patch MMR and short patch Vsr endonuclease and to be mediated by MutL. ${ }^{86}$ It is also possible that MLH1 facilitates binding of MED1 to DNA, similar to MutL stimulation of Vsr and MutS DNA binding.

Finally, the role of MLH1 in MED1 repair reactions may involve interplay with DNA methylation, as a distinguishing feature of MED1 in comparison to TDG is the presence of the MBD domain. Since the MBD and methylation of the mismatched $\mathrm{CpG}$ sites are not required for efficient catalysis by MED1, ${ }^{78,81}$ the MBD is involved in some other function perhaps integrating the epigenetic signal of cytosine methylation with MED1- or MLH1-mediated transactions. One possibility is that the MBD facilitates localization of MED1 to regions of the genome rich in methylated CpG sites. $^{78,80}$

\section{Role and interactions of MMR components in recombination and meiosis}

In bacteria and yeast, MMR is involved in suppression of homologous recombination between related but slightly divergent ('homeologous') sequences, thus raising a genetic barrier that prevents interspecies gene transfer. ${ }^{2,87}$ It is possible that during suppression of homeologous recombination, MMR proteins recognize DNA heterologies, mispairs and 
perturbed DNA structures, such as Holliday junctions, in a manner similar to mismatch recognition. Then, activation of subsequent downstream events would be specific for the recombinational repair pathway. ${ }^{88}$

In yeast, binding of Msh factors would in itself abort the recombination reaction whereas Mlh factors may play a lesser role in recombination. ${ }^{88}$ Alternatively, rejection of homeologous recombination may occur via the Msh2Msh3-mediated recruitment of either endonucleases/exonucleases or helicases, which would respectively degrade or unwind the recombination intermediate. ${ }^{88}$ The Msh2Msh3 complex does recruit the endonuclease Rad1Rad10 (homologue of the human NER factor, XPFERCC1) for removal of the non-homologous $3^{\prime}$ tails ${ }^{89}$ (Figure 3). The Msh2-interactor, Exonuclease I, also displays anti-recombinogenic activity. Finally, the yeast Sgs1 helicase, homologue of the Bloom and Werner syndrome proteins, BLM and WRN, acts in the same epistatic group with MMR proteins in suppressing homeologous recombinations as well as gross chromosomal rearrangements. ${ }^{90}$

Interestingly, in two budding yeast species, defects in MMR promote homeologous recombination at telomeric and subtelomeric sequences, maintaining telomere length in the absence of telomerase activity, an effect that might also occur in MMR-defective tumors. ${ }^{91}$

The molecular mechanisms underlying suppression of genetic recombination by MMR in mammalian cells are less clear and the relevant interactions with recombinationspecific factors are not established. Recently, the human Bloom syndrome helicase BLM, a component of BASC, was shown to directly interact with MLH1 (Figure 3), ${ }^{92}$ lending support to the above mentioned genetic interaction detected in yeast between Sgs1 and Msh2. ${ }^{90}$ MMR does appear to suppress somatic recombination in mammalian cells. Thus, a human HeLa subclone lacking expression of PMS2 was found to have an increased rate of somatic recombination between two copies of a selectable gene, in comparison to the MMR-proficient parental cell line. ${ }^{93}$ Similarly, increased somatic recombination has been reported in Msh2-null mouse ES cells. ${ }^{94}$ Lymphomas in Msh2-null mice may arise through chromosomal aberrations caused by this hyper-recombinogenic phenotype. Based on these findings, it is indeed surprising that MMR-deficient human tumors usually exhibit a diploid or near-diploid karyotype. ${ }^{24}$

In addition to a role in somatic recombination, MMR proteins are involved in the regulation of meiotic recombination. In this area, the initial observations were conducted in yeast. Fidelity of chromosome segregation requires both sister chromatid cohesion and meiotic crossing-over of homologous chromosomes. ${ }^{95}$ Yeast mutants defective in Mlh1 and MIh3 show reduction in meiotic crossing-over and this effect is epistatic with Msh4 and Msh5 mutations, thus indicating that the heterodimers Msh4-Msh5 and Mlh1-Mlh3 function during meiotic recombination ${ }^{25}$ (Figure 1). No meiotic phenotype was observed for yeast MIh2 and Pms1 mutants. ${ }^{25}$

In keeping with the meiotic phenotype of their yeast homologues, $\mathrm{Mlh}^{-1-}, \mathrm{Msh}^{-1-}$ and $\mathrm{Msh}^{-1-}$ male and female mice are sterile; and unlike yeast Pms1 mutants, $P m s 2^{-1-}$ mice exhibit male infertility. ${ }^{96-99}$ In MIh1-null mice, chromosomal pairing is initially normal and sterility is the consequence of reduced crossing-over. ${ }^{97}$ In normal spermatocytes, Mlh1 protein localizes at chiasmata, the points where crossing-over occurs; in oocytes, Mlh1 localizes at the synaptonemal complex and at chiasmata. In MIh1-null mice, chiasmata in male and female meiosis are reduced by 1-2 orders of magnitude and homologous chromosomes are frequently separated. ${ }^{97}$ These findings clearly establish a role for Mlh1 in chiasma formation and stabilization.

Male sterility in $P m s 2^{-1-}$ mice is associated with defects in chromosomal synapsis indicating a requirement for Pms2 at an earlier stage than Mlh1. ${ }^{96}$ The meiotic defect in male and female $\mathrm{Msh}^{-1-}$ and $M s h 5^{-1-}$ mice is similar, in that chromosomes fail to undergo normal pairing during zygonema, consistent with their coordinate obligate role at the same time in meiosis. ${ }^{98,99}$ Based on the above findings, it would be interesting to ascertain also whether MIh3-null mice are sterile and exhibit a meiotic phenotype.

Mechanistically, it has been hypothesized that MSH4MSH5 and MLH1-MLH3 complexes promote the formation and stabilization of Holliday junctions and their preferential resolution into cross-overs rather than noncross-overs. ${ }^{100}$ Apoptosis was observed in Mlh1-null, Msh4-null and Msh5null mouse testes, suggesting that abnormal meiosis activates a checkpoint leading to cell death via Msh4Msh5 and Mlh1-Mlh3 complexes. ${ }^{100}$ This implies coordination between DNA perturbation and MMR-mediated regulation of apoptosis. This is an emerging function of MMR proteins, not only in meiotic cells but also in mitotic cells (see below).

A schematic of the multiple interactions of MMR proteins in TCR, BER and recombination is shown in Figure 3.

\section{Signaling aspects of MMR proteins in sensing DNA damage and promoting apoptosis}

An important function of MMR proteins is to sense DNA damage and mediate the induction of apoptosis. Although this process is still poorly understood and the relevant physical interactions involved are not known in detail, a network of functional and signaling interactions stemming from MMR proteins is taking shape.

\section{MMR and DNA damage: response to alkylating agents, cytotoxicity and cell cycle checkpoints}

Cytotoxicity of some alkylating agents and some DNA damaging and anti-cancer agents requires a functional MMR. ${ }^{101,102} N$-methyl- $N$ '-nitro- $N$-nitrosoguanidine (MNNG), $N$-methyl- $N$-nitrosourea (MNU) and their analogues used in the clinic, temozolomide and dacarbazine, cause the same DNA damage, methylation of the $O^{6}$ position of guanine in DNA to form $O^{6}$-methylguanine $\left(O^{6}-\mathrm{meG}\right)$. $O^{6}-\mathrm{meG}$ can be inactivated by the suicidal enzyme $O^{6}$-meG methyltransferase. However, it is the presence of a functional MMR system 
which mediates $O^{6}-$ meG cytotoxicity. The observation that MMR-deficient $E$. coli strains are resistant to killing by MNNG and MNU was soon extended to eukaryotic cells: induction of resistance to these agents is associated with loss of expression or function of MMR genes, particularly in the absence of $O^{6}$-meG methyltransferase. ${ }^{101,102}$ Similarly, cell lines defective in MSH2, MSH6, MLH1 and PMS2 are resistant to alkylating agents cytotoxicity. Thus, the persistence in DNA of $0^{6}$-meG causes cytotoxicity in an MMRdependent fashion: in the absence of a functional MMR, DNA damage accumulates but does not trigger cell death. For this reason, the accumulation of $O^{6}$-meG in MMR-deficient cells has been named alkylation or methylation 'tolerance', a more appropriate term than resistance. Tolerance to $O^{6}-\mathrm{meG}$ is associated with cross-tolerance to 6-thioguanine (6-TG), another antiproliferative agent used in the clinic. The methyldonor S-adenosylmethionine methylates 6-TG to form $S^{6}$ methylthioguanine $\left(S^{6}-\mathrm{meG}\right)$ in a non-enzymatic reaction.

The role of MMR in tolerance to alkylating agents can be explained by the recognition and binding of $O^{6}-$ meG and $S^{6}-\mathrm{meG}$ by hMutS $\alpha$. In addition, both $O^{6}-\mathrm{meG}$ and $S^{6}$ meG can direct misincorporation of $T$ during DNA synthesis. The resulting $O^{6}-$ meG:T and $S^{6}-$ meG:T mismatches are recognized even more efficiently by MMR, and $0^{6}$-meG:T effectively stimulates the ATPase activity of the MSH2-MSH6 heterodimer.

Interestingly, cytotoxicity of alkylating agents is associated with a $\mathrm{G}_{2}-\mathrm{M}$ cell cycle checkpoint. MNNG or 6-TG treatment induces a $G_{2}$ cell cycle arrest in cell lines harboring a functional MMR, in keeping with the postreplicative nature of this repair system. To the contrary, upon alkylating agent exposure, MMR-deficient cell lines are able to go through the $\mathrm{G}_{2}-\mathrm{M}$ checkpoint and arrest in $\mathrm{G}_{1}$, the secondary cell cycle hit of $6-T \mathrm{G}^{103,104}$ Thus, the defective $\mathrm{G}_{2}-\mathrm{M}$ checkpoint induced by $6-\mathrm{TG}$ exposure of MLH1-defective human colorectal HCT-116 cells and of Mlh1-deficient mouse embryonic fibroblasts (MEFs) can be restored by transfer of human chromosome 3 and expression of the human $\mathrm{MLH1}$ gene, respectively. ${ }^{103,104}$ In Mlh1-deficient MEFs, within one day of restoration of the $\mathrm{G}_{2}-\mathrm{M}$ checkpoint by expression of human $\mathrm{MLH1}$, the cell cycle profile showed a possibly apoptotic, pre-G1 peak. ${ }^{104}$

\section{MMR and induction of apoptosis: implications for tumorigenesis}

What is the relationship between alkylating agents, MMR and induction of cell death? Traditionally, two models have been proposed, which at the present status of knowledge are not necessarily mutually exclusive: the model of futile cycles of repair and the model of direct signaling of apoptosis.

In the first model, initially proposed for $E$. coli, MMR of mismatches containing $O^{6}-$ meG or $S^{6}-$ meG leads to the removal of the paired $\mathrm{C}$ or $\mathrm{T}$ in the newly synthesized strand. However, persistence of the alkylated base in the template strand promotes additional futile cycles of excision and resynthesis, ultimately leading to replication fork delays, strand breaks and gaps, which in turn induce cell death. This model explains the observations that cell death and the parallel induction of chromosomal rearrangements occur only in the second cell cycle after treatment with the alkylating agent. ${ }^{105}$

In the second model, recognition of DNA damage by MMR proteins initiates a signal transduction cascade directly activating the cell cycle checkpoint and engaging the apoptotic machinery. ${ }^{35,64,103}$ This model is compatible with the finding that, in MMR-proficient and -deficient cell lines, overexpression of $\mathrm{MSH} 2$ and $\mathrm{MLH} 1$, but not $\mathrm{MSH}$, MSH3 and PMS2, induces apoptosis, ${ }^{106}$ further highlighting the primary signaling properties of $\mathrm{MSH} 2$ and $\mathrm{MLH} 1$. Indeed, this model represents an extension of the concept of MMR proteins as molecular switches. ${ }^{33}$

Both models have an important implication, in that they provide an explanation for the selection/growth advantage of cells harboring MMR gene mutations during tumorigenesis: in addition to the acquisition of a mutator phenotype, inactivation of MMR may endow tumor cells with a reduced apoptotic response to the DNA damage elicited in the gut by exogenous (food-borne) or endogenous mutagens and alkylating agents. ${ }^{35,106}$ In this context, subsequent accumulation of frameshift inactivating mutations in the proapoptotic genes $B A X$ and, possibly, caspase-5 may give tumor cells an additional growth advantage.

\section{Involvement of p53-dependent and -independent mechanisms of apoptosis}

What are the molecular determinants of MMR-provoked induction of apoptosis? The tumor suppressor protein p53 is a classic transducer of the apoptotic signal of DNA damage and environmental stress. ${ }^{107}$ Activation of p53 by these stimuli leads to its phosphorylation, stabilization and transcriptional modulation (both activation and repression) of p53target genes. ${ }^{107}$ In addition, p53 regulates cell cycle progression by controlling the $\mathrm{G}_{1}-\mathrm{S}$ and $\mathrm{G}_{2}-\mathrm{M}$ checkpoints. Thus, p53 is an obvious candidate for mediating the MMRdependent apoptotic response to alkylating agents.

Indeed, p53 is phosphorylated on serine residues 15 and 392 in response to MNU and MNNG alkylation damage. ${ }^{108}$ These phosphorylation events, which accompany an increase in p53 protein levels, are dependent on functional hMutS $\alpha$ and hMutL $\alpha$ and independent of hMutS $\beta .^{108}$

Despite these observations, several studies rule out a necessary role of p53 in the apoptosis induced by methylation damage. Thus, induction of apoptosis in human lymphoblastoid cells treated with MNNG is dependent upon functional hMutS $\alpha$; interestingly, in the same system, stabilization of p53 is also dependent upon functional hMutS $\alpha{ }^{109}$ However, when p53 expression was abrogated by transfection of the human papilloma virus 16 E6 gene, MNNG-induced apoptosis was not affected, as it would be expected for a p53-independent process. ${ }^{109}$

How can the p53-independent nature of alkylating agentinduced apoptosis be reconciled with the MMR-dependent phenomena of p53 phosphorylation on serines 15 and 392 and protein stabilization? It has been suggested that p53 plays two separate roles in the cellular response to DNAdamaging agents, i.e. control of cell cycle checkpoints and regulation of apoptosis. ${ }^{110}$ Thus, it is possible that Ser-15/ Ser-392 phosphorylation and stabilization of p53 in 
response to alkylating agents are part of pathway(s) initiated by MMR proteins as general sensors of DNA damage; such pathway(s) would impinge on cell cycle checkpoints but not on apoptosis. Indeed, it is p53 phosphorylation on Ser-46 which plays a pivotal role in p53-dependent apoptosis. ${ }^{111}$ The kinase phosphorylating p53 on Ser-46 might be p38. ATM and ATR might phosphorylate p53 on Ser-15, whereas Ser-392 is a possible target of casein kinase II and p38 (Figure 4).

\section{The role of Abl and p73 in MMR-dependent apoptosis}

The lack of a primary involvement of p53 in MMR-dependent apoptosis is not totally unexpected since this cell fate regulator is mostly involved in signaling apoptosis following double-strand DNA breaks. ${ }^{112}$ It is likely that the definitive proof ruling out the necessary involvement of p53 in MMRregulated apoptosis will come from studies of cell death upon alkylating agent treatment of Tp53-null mouse embryo fibroblasts or ES cells.

In the absence of a necessary role of p53, what are the mediators of MMR-dependent apoptosis? In addition to alkylation damage, MMR modulates sensitivity to other DNA damaging molecules, such as cisplatin, a chemotherapeutic agent which forms intra- and interstrand adducts; the topoisomerase inhibitors, doxorubicin and etoposide; the antimetabolite, 5-fluorouracil; and ionizing radiations. ${ }^{113}$ The degree of resistance/tolerance to these molecules afforded by defective MMR is less pronounced than that towards alkylating agents. ${ }^{105}$ It has been suggested that this is the result of a common role of MMR proteins in signaling DNA perturbations combined with a direct role in the repair of the lesions induced by these molecules, which would be less prominent in comparisons to a major role in the repair of alkylation damage. ${ }^{105}$ Nevertheless, it has been the analysis of the role of MMR in cisplatin-induced damage which recently disclosed an essential role of the oncoprotein $\mathrm{Abl}$ and the p53-related protein p73 in MMRdependent, p53-independent apoptosis.

Cisplatin forms bifunctional covalent intrastrand DNA adducts by reacting with the $N^{7}$ atoms of purines at GpG, $A p G$ and $\mathrm{GpNpG}$ sequences, in order of decreasing frequency. hMutS $\alpha$ can bind to the cisplatin GpG adduct and to DNA containing a $T$ opposite the $3^{\prime}$ platinated $\mathrm{G}^{64}$ Binding by hMut $\alpha$ is likely a reflection of the fact that the cisplatin-modified DNA is kinked towards the major groove much like mismatched DNA (see above). Loss of MMR function due to hMutS $\alpha$ or hMutL $\alpha$ defects is associated with low-level (approximately twofold) resistance to cisplatin and the related compound carboplatin. Acquisition of this low-level resistance is likely to be clinically relevant in determining failure of platinum-based chemotherapy.

As for alkylating agents, cisplatin resistance caused by loss of MMR function is likely to result from a tolerance mechanism, i.e. from the inability to detect DNA adducts and activate signal transduction pathways leading to apoptosis. One of these pathways involves the oncogenic tyrosine kinase Abl. Cisplatin and other DNA-damaging agents such as ionizing radiation and mitomycin- $C$ can activate the Abl kinase activity, ${ }^{114,115}$ resulting in cell cycle arrest and apoptosis. ${ }^{114}$ MLH1-defective HCT-116 cells and

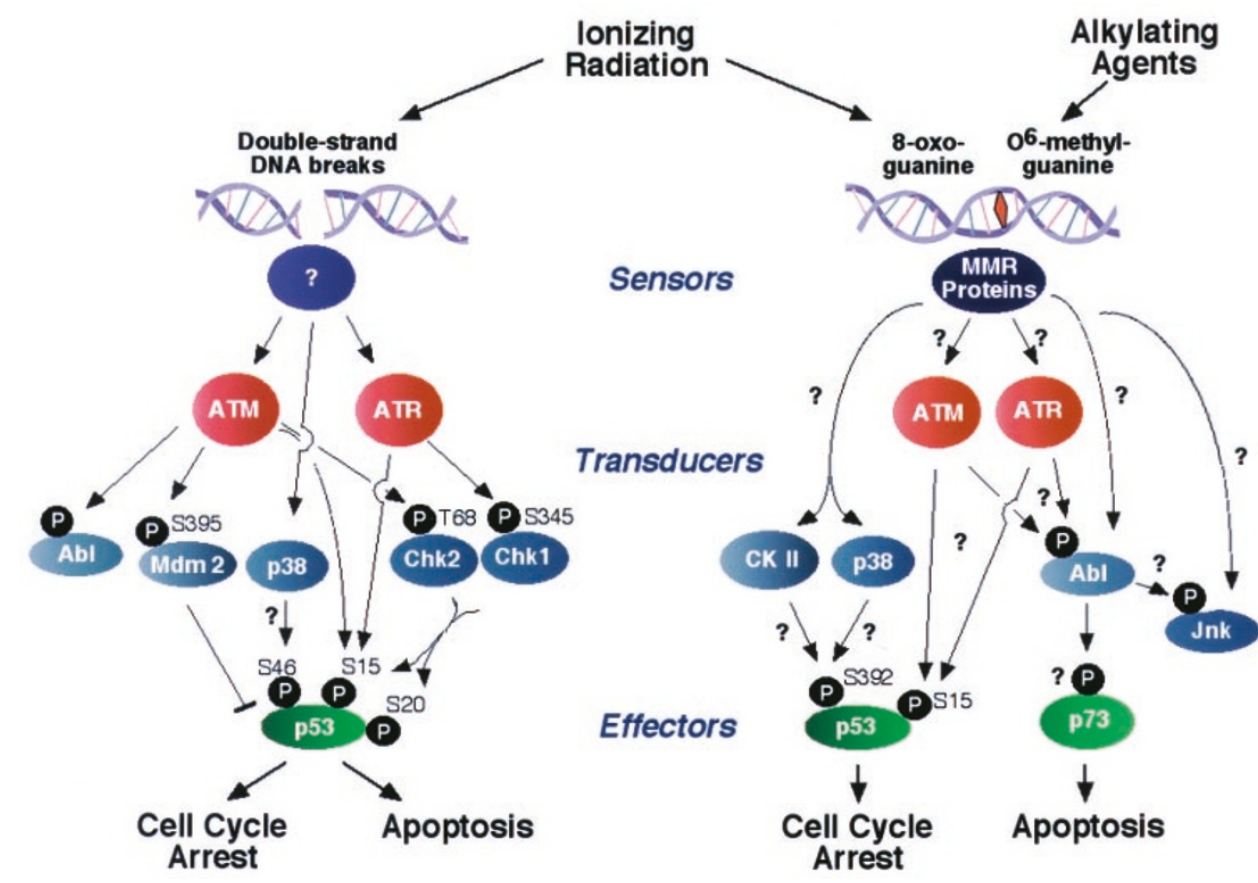

Figure 4 Cell cycle and apoptosis signaling pathways initiated by DNA damage. Ionizing radiation (or cisplatin) activates both MMR-independent and -dependent pathways, whereas alkylating agents only function through the latter mechanism. The MMR-dependent pathway activates both p53-dependent cell cycle checkpoint and p73-dependent apoptosis. In the MMR-dependent pathway, the precise involvement of the indicated signaling molecules is not well characterized. CKII: casein kinase II. See text for details 
MSH2-defective HEC-59 cells fail to activate Abl upon cisplatin treatment. ${ }^{115,116}$ Upon restoration of a functional MMR by transfer of chromosome 3 to HCT-116 cells and of chromosome 2 to HEC-59 cells, cisplatin activation of $\mathrm{Abl}$ kinase activity is reestablished. ${ }^{115,116}$

MMR proficiency is also associated with increased halflife and consequent stabilization of the protein p73 following cisplatin treatment, an effect that can be mimicked by cotransfection of kinase-active Abl. ${ }^{116}$ These experiments establish p73, a p53-related protein that when overexpressed is pro-apoptotic, as a key mediator of MMRdependent, p53-independent apoptosis caused by cisplatin. Indeed, cotransfection with Abl potentiates the pro-apoptotic function of p73 but not p53. ${ }^{116}$ Even more cogently, in Mlh1-null and Abl-null mouse embryo fibroblasts (MEFs), but not in wild-type or Tp53-null MEFs, induction of p73 by cisplatin is abrogated, thus confirming that functional MMR and $\mathrm{Abl}$ are necessary and p53 is dispensable for p73 response to cisplatin. ${ }^{116}$ As expected, MIh1-null and $\mathrm{Ab} /$ null MEFs show reduced sensitivity to cisplatin killing. ${ }^{116}$

These findings outline a p53-independent apoptotic pathway initiated by cisplatin-induced DNA damage and mediated by MMR detection of DNA perturbation, Abl kinase activation and p73 stabilization (Figure 4). The mechanistic details of this pathway are still not clear. The link between platinated DNA damage detection and Abl kinase activation has yet to be established. Perhaps, the existence of a single multimeric complex of MMR proteins and $A T M,{ }^{67}$ an upstream effector of $A b l$, explains this issue. Similarly, the relationship between activated Abl and p73 stabilization is unclear; it has been reported that $\mathrm{Abl}$ and p73 physically interact, but tyrosine phosphorylation of p73 in response to cisplatin was not detected. ${ }^{116}$ However, Abl was shown to phosphorylate p73 in vivo on tyrosine 99 in response to ionizing radiation. In addition, it has been described that the serine threonine kinase JNK is activated in response to cisplatin treatment in a MMR-dependent fashion; ${ }^{115}$ it is unclear if JNK activation is independent of Abl but it does not seem to be linked to apoptosis.

In summary, MMR-dependent apoptosis is mediated by stabilization of p73, whereas the MMR-dependent induction of p53 does not elicit apoptosis and may only affect cell cycle checkpoint activation (Figure 4). It would be important to confirm that the MMR-dependent activation of $\mathrm{Abl}$ and p73 is not limited to cisplatin but also occurs in response to DNA damage primarily detected and repaired by MMR, namely that induced by alkylating agents.

Furthermore, it should be recognized that DNA damaging agents that engage multiple sensors might give rise to a complex apoptotic response. Thus, cisplatin induces parallel apoptotic pathways by activating both p73 in a MMR-dependent fashion (as described above) and p53 in a MMR-independent fashion. ${ }^{116}$ Similarly, ionizing radiation induces apoptosis via both p53-dependent and MMRdependent (presumably p73-dependent) pathways. These two pathways are parallel and do not significantly crosstalk, giving rise to an additive effect ${ }^{117}$ (Figure 4). For ionizing radiation, the pathway of p53-mediated apoptosis is likely to depend on the induction of single- and doublestrand DNA breaks; to the contrary, the MMR-dependent apoptosis is likely caused by the binding of MMR complexes to 8-oxoguanine generated by ionizing radiation $^{117}$ (Figure 4). Incidentally, based on this consideration, the model of the direct signaling properties of MMR proteins might be preferred over that of futile cycles of repair, as the latter implies the presence of DNA strand breaks.

Certainly, additional studies are warranted in this area, as deviations from the general scheme described in Figure 4 have been described. Thus, p53-dependent and p53independent apoptosis mediated by Msh2 has been described in vivo, in small intestine crypts of mice treated with alkylating agents and cisplatin. ${ }^{118}$ Also, DNA adducts, formed by some carcinogenic aromatic amines appear to induce MMR-dependent apoptosis by both p53-dependent and p53-independent mechanisms. ${ }^{119}$

\section{Conclusion and future perspectives}

In 1993 MMR entered the center stage of cancer research and molecular medicine with the seminal discoveries of its role in the pathogenesis of HNPCC and resistance/tolerance to alkylating agents and other antineoplastic drugs. After 8 years of intense investigation in laboratories around the world, many aspects of the genetics, biochemistry, cell biology and pharmacology of MMR have become established 'textbook' concepts in biology and medicine.

It is now clear that MMR proteins are engaged in a complex network of molecular interactions that extends to proteins participating in other DNA transactions, such as recombination, transcription-coupled repair and base excision repair. MMR proteins perform the daunting task of repairing an ephemeral DNA lesion, the mismatch, which is formed by chemically normal bases and would disappear in the next round of DNA replication. On the other hand, excessive damage beyond a critical threshold activates apoptosis in an MMR-dependent fashion. Thus, the complexity of the interactions of MMR components is a reflection of their central role in mutation avoidance and cell death regulation. Indeed, inactivating mutations of MMR genes in hereditary and sporadic cancer lead to both an increase of the mutation rate and inhibition of apoptosis.

In the future, efforts should be directed towards novel molecular approaches to cancer prevention and treatment that build on our increased understanding of MMR function. Chemopreventive agents should be developed that specifically target precancerous cells in which an incipient defect in MMR is propelling them to malignancy. Genetic selection for microsatellite stability in MMR-defective cells by the nonsteroidal anti-inflammatory drugs aspirin and sulindac $^{120}$ offers concrete hope that this approach might be effective. At the same time, novel radio- and chemotherapeutic strategies will have to take into account the MMR proficiency or deficiency of the individual tumor. Whereas MMR-proficient tumors are likely to respond to conventional alkylating and DNA damaging agents, MMR deficient malignancies could be treated preferentially with frameshift-inducing agents and other drugs for which increased sensitivity is brought about by a MMR defect.

It is likely that pharmacological exploitation of the numerous interactions of MMR proteins will prove fruitful. 
For instance, acquisition of resistance/tolerance to conventional anti-neoplastic therapy by mutations in MMR genes could be circumvented by novel molecules that restore the pro-apoptotic circuitry downstream of the faulty DNA damage sensors. Similarly, it might be useful to selectively inhibit some but not all the extra-MMR interactions: inhibition of the TCR capacity of $\mathrm{MSH} 2$ without affecting its role in cell death might sensitize cells to oxidative damage.

Finally, eukaryotic MMR still has to disclose its most fascinating secret, the molecular determinants of strand specificity. I am confident that understanding this process in the future will lead to a deeper comprehension of fundamental features of DNA replication and repair, and will open new strategies of targeted cancer drug design.

\section{Acknowledgements}

The author wishes to thank Dr. Wei Yang for the kind gift of the 'induced fit' schematic that was modified for Figure $2 \mathrm{C}$ with permission from Cold Spring Harbor Press; Drs. Jim Drummond, Maureen Murphy and Robert Perry for helpful comments; Drs. Yoshihiro Matsumoto, Steven Seeholzer and Anthony Yeung and Mr. John Upson for critical reading of the manuscript; Mr. Amar Majmundar for help with Figures 3 and 4; and Rose Sonlin for secretarial assistance. The author apologizes to colleagues for having failed to cite their work due to space constraints. This study was supported by NIH grants CA78412, and CA06927. Additional support was provided by an appropriation from the Commonwealth of Pennsylvania to the Fox Chase Cancer Center.

\section{References}

1. Fishel $R$ and Kolodner RD (1995) Identification of mismatch repair genes and their role in the development of cancer. Curr. Opin. Genet. Dev. 5: 382

2. Modrich $P$ and Lahue R (1996) Mismatch repair in replication fidelity, genetic recombination, and cancer biology. Annu. Rev. Biochem. 65: 101-133

3. Jiricny J (1998) Replication errors: cha(lle)nging the genome. EMBO J. 17: 6427-6436

4. Kolodner RD and Marsischky GT (1999) Eukaryotic DNA mismatch repair. Curr. Opin. Genet. Dev. 9: 89-96

5. Kinzler KW and Vogelstein B (1996) Lessons from hereditary colorectal cancer. Cell 87: $159-170$

6. Loeb LA (1998) Cancer cells exhibit a mutator phenotype. Adv. Cancer Res. 72: $25-56$

7. Lynch HT and de la Chapelle A (1999) Genetic susceptibility to non-polyposis colorectal cancer. J. Med. Genet. 36: 801-818

8. Perucho M (1996) Cancer of the microsatellite mutator phenotype. Biol. Chem. 377: $675-684$

9. Burdett V, Baitinger C, Viswanathan M, Lovett ST and Modrich P (2001) In vivo requirement for RecJ, ExoVII, Exol, and ExoX in methyl-directed mismatch repair. Proc. Natl. Acad. Sci. USA 98: 6765-6770

10. Drummond JT, Li GM, Longley MJ and Modrich P (1995) Isolation of an hMSH2p160 heterodimer that restores DNA mismatch repair to tumor cells. Science 268: 1909-1912

11. Palombo F, laccarino I, Nakajima E, Ikejima M, Shimada T and Jiricny J (1996) $\mathrm{hMutS} \beta$, a heterodimer of $\mathrm{hMSH} 2$ and $\mathrm{hMSH} 3$, binds to insertion/deletion loops in DNA. Curr. Biol. 6: 1181-1184

12. Bocker T, Barusevicius A, Snowden T, Rasio D, Guerrette S, Robbins D, Schmidt C, Burczak J, Croce CM, Copeland T, Kovatich AJ and Fishel R (1999) hMSH5: a human MutS homologue that forms a novel heterodimer with hMSH4 and is expressed during spermatogenesis. Cancer Res. 59: 816-822
13. Li GM and Modrich $P$ (1995) Restoration of mismatch repair to nuclear extracts of $\mathrm{H} 6$ colorectal tumor cells by a heterodimer of human MutL homologs. Proc. Natl. Acad. Sci. USA 92: 1950-1954

14. Raschle M, Marra G, Nystrom-Lahti M, Schar P and Jiricny J (1999) Identification of hMutLbeta, a heterodimer of hMLH1 and hPMS1. J. Biol. Chem. 274: 32368-32375

15. Lipkin SM, Wang V, Jacoby R, Banerjee-Basu S, Baxevanis AD, Lynch HT, ElliottRM and Collins FS (2000) MLH3: a DNA mismatch repair gene associated with mammalian microsatellite instability. Nat. Genet. 24: 27-35

16. Littman SJ, Fang WH and Modrich P (1999) Repair of large insertion/deletion heterologies in human nuclear extracts is directed by a $5^{\prime}$ single-strand break and is independent of the mismatch repair system. J. Biol. Chem. 274: 74747481

17. Umar A, Risinger JI, Glaab WE, Tindall KR, Barrett JC and Kunkel TA (1998) Functional overlap in mismatch repair by human MSH3 and MSH6. Genetics 148: $1637-1646$

18. Obmolova G, Ban C, Hsieh P and Yang W (2000) Crystal structures of mismatch repair protein MutS and its complex with a substrate DNA. Nature 407: 703-710

19. Haber LT and Walker GC (1991) Altering the conserved nucleotide binding motif in the Salmonella typhimurium MutS mismatch repair protein affects both its ATPase and mismatch binding activities. EMBO J. 10: 2707-2715

20. Allen DJ, Makhov A, Grilley M, Taylor J, Thresher R, Modrich P and Griffith JD (1997) MutS mediates heteroduplex loop formation by a translocation mechanism. EMBO J. 16: 4467-4476

21. Ban C and Yang W (1998) Crystal structure and ATPase activity of MutL: implications for DNA repair and mutagenesis. Cell 95: 541-552

22. Prolla TA, Pang Q, Alani E, Kolodner RD and Liskay RM (1994) MLH1, PMS1, and MSH2 interactions during the initiation of DNA mismatch repair in yeast. Science 265: 1091-1093

23. Gu L, Hong Y, McCulloch S, Watanabe H and Li GM (1998) ATP-dependent interaction of human mismatch repair proteins and dual role of PCNA in mismatch repair. Nucleic Acids Res. 26: 1173-1178

24. Buermeyer AB, Deschenes SM, Baker SM and Liskay RM (1999) Mammalian DNA mismatch repair. Annu. Rev. Genet. 33: 533-564

25. Wang TF, Kleckner N and Hunter N (1999) Functional specificity of MutL homologs in yeast: evidence for three Mlh1-based heterocomplexes with distinct roles during meiosis in recombination and mismatch correction. Proc. Natl. Acad. Sci. USA 96: 13914-13919

26. Lamers MH, Perrakis A, Enzlin JH, Winterwerp HH, de Wind N and Sixma TK (2000) The crystal structure of DNA mismatch repair protein MutS binding to a $G$ T mismatch. Nature 407: 711-717

27. Kolodner RD (2000) Guarding against mutation. Nature 407: 687, 689

28. Junop MS, Obmolova G, Rausch K, Hsieh P and Yang W (2001) Composite active site of an ABC ATPase: MutS uses ATP to verify mismatch recognition and authorize DNA repair. Mol. Cell. 7: 1-12

29. Gradia S, Subramanian D, Wilson T, Acharya S, Makhov A, Griffith J and Fishel R (1999) hMSH2-hMSH6 forms a hydrolysis-independent sliding clamp on mismatched DNA. Mol. Cell. 3: 255-261

30. Hopfner K-P and Tainer JA (2000) DNA mismatch repair: The hands of a genome guardian. Structure 8: R237-R241

31. Ban C, Junop M and Yang W (1999) Transformation of MutL by ATP binding and hydrolysis: a switch in DNA mismatch repair. Cell 97: 85-97

32. Ban C and Yang W (1998) Structural basis for MutH activation in E.coli mismatch repair and relationship of MutH to restriction endonucleases. EMBO J. 17: $1526-1534$

33. Fishel R (1998) Mismatch repair, molecular switches, and signal transduction. Genes Dev. 12: 2096-2101

34. Gradia S, Acharya S and Fishel R (1997) The human mismatch recognition complex hMSH2-hMSH6 functions as a novel molecular switch. Cell 91: 9951005

35. Fishel R (1999) Signaling mismatch repair in cancer. Nat. Med. 5: 1239-1241

36. Blackwell LJ, Martik D, Bjornson KP, Bjornson ES and Modrich P (1998) Nucleotide-promoted release of hMutS $\alpha$ from heteroduplex DNA is consistent with an ATP-dependent translocation mechanism. J. Biol. Chem. 273: 3205532062

37. Galio L, Bouquet $C$ and Brooks $P$ (1999) ATP hydrolysis-dependent formation of a dynamic ternary nucleoprotein complex with MutS and MutL. Nucleic Acids Res. 27: $2325-2331$ 
38. Habraken Y, SungP,PrakashLandPrakashS(1998)ATP-dependentassembly of a ternary complex consisting of a DNA mismatch and the yeast MSH2-MSH6 and MLH1-PMS1 protein complexes. J. Biol. Chem. 273: 9837-9841

39. Schofield MJ, Nayak S, Scott TH, Du C and Hsieh P (2001) Interaction of MutS and MutL at a DNA mismatch. J. Biol. Chem. 176: 28291-28299

40. Jiricny J (2000) Mismatch repair: The praying hands of fidelity. Curr. Biol. 10: R788-R790

41. Sixma TK (2001) DNA mismatch repair: MutS structures bound to mismatches. Curr. Opin. Struct. Biol. 11: 47-52

42. Umar A, Buermeyer AB, Simon JA, Thomas DC, Clark AB, Liskay RM and Kunkel TA (1996) Requirement for PCNA in DNA mismatch repair at a step preceding DNA resynthesis. Cell 87: 65-73

43. Kokoska RJ, Stefanovic L, Buermeyer AB, Liskay RM and Petes TD (1999) A mutation of the yeast gene encoding PCNA destabilizes both microsatellite and minisatellite DNA sequences. Genetics 151: 511-519

44. Clark AB, Valle F, Drotschmann K, Gary RK and Kunkel TA (2000) Functional interaction of PCNA with MSH2-MSH6 and MSH2-MSH3 complexes. J. Biol. Chem. 275: 36498-36501

45. Flores-Rozas H, Clark D and Kolodner RD (2000) Proliferating cell nuclear antigen and Msh2p-Msh6p interact to form an active mispair recognition complex. Nat. Genet. 26: 375-378

46. KleczkowskaHE,Marra G, LettieriT and JiricnyJ(2001)hMSH3 and hMSH6 interact with PCNA and colocalize with it to replication foci. Genes Dev. 15: 724-736

47. Bowers J, Tran PT, Joshi A, Liskay RM and Alani E (2001) MSH-MLH complexes formed at a DNA mismatch are disrupted by the PCNA sliding clamp. J. Mol. Biol. 306: 957-968

48. Schmutte C, Marinescu RC, Sadoff MM, Guerrette S, Overhauser J and Fishel R (1998) Human exonuclease linteracts with the mismatch repair protein hMSH2. Cancer Res. 58: 4537-4542

49. Wu Y, Berends MJ, Post JG, Mensink RG, Verlind E, Van Der Sluis T, Kempinga C, Sijmons RH, Van Der Zee AG, Hollema H, Kleibeuker JH, Buys $\mathrm{CH}$ and Hofstra RM (2001) Germline mutations of exo1 gene in patients with hereditary nonpolyposis colorectal cancer (hnpcc) and atypical hnpcc forms. Gastroenterology 120: $1580-1587$

50. Modrich $\mathrm{P}$ (1997) Strand-specific mismatch repair in mammalian cells. J. Biol. Chem. 272: 24727-24730

51. Boland CR (2000) Molecular genetics of hereditary nonpolyposis colorectal cancer. Ann. NY Acad. Sci. 910: 50-61

52. Boland CR, Thibodeau SN, Hamilton SR, Sidransky D, Eshleman JR, Burt RW, Meltzer SJ, Rodriguez-Bigas MA, Fodde R, Ranzani GN and Srivastava S (1998) A National Cancer Institute Workshop on Microsatellite Instability for cancer detection and familial predisposition: development of international criteria for the determination of microsatellite instability in colorectal cancer. Cancer Res. 58: 5248-5257

53. DietmaierW, Wallinger S, Bocker T, Kullmann F, Fishel R and RuschoffJ (1997) Diagnostic microsatellite instability: definition and correlation with mismatch repair protein expression. Cancer Res. 57: 4749-4756

54. Guerrette S, Acharya S and Fishel R (1999) The interaction of the human MutL homologues in hereditary nonpolyposis colon cancer. J. Biol. Chem. 274 $6336-6341$

55. Guerrette S, Wilson T, Gradia S and Fishel R (1998) Interactions of human $\mathrm{hMSH} 2$ with $\mathrm{hMSH} 3$ and $\mathrm{hMSH} 2$ with $\mathrm{hMSH} 6$ : examination of mutations found in hereditary nonpolyposis colorectal cancer. Mol. Cell. Biol. 18: 6616-6623

56. Lindahl T and Wood RD (1999) Quality control by DNA repair. Science 286 $1897-1905$

57. Sancar A (1994) Mechanisms of DNA excision repair. Science 266: 1954 - 1956

58. Hanawalt PC (1994) Transcription-coupled repair and human disease. Science 266: $1957-1958$

59. Leadon SA (1999) Transcription-coupled repair of DNA damage: unanticipated players, unexpected complexities. Am. J. Hum. Genet. 64: 1259-1263

60. Mellon I, Rajpal DK, Koi M, Boland CR and Champe GN (1996) Transcriptioncoupled repair deficiency and mutations in human mismatch repair genes. Science 272: $557-560$

61. Leadon SA and Avrutskaya AV (1997) Differential involvement of the human mismatch repair proteins, hMLH1 and hMSH2, in transcription-coupled repair Cancer Res. 57: 3784-3791

62. Le Page F, Randrianarison V, Marot D, Cabannes J, Perricaudet M, Feunteun J and Sarasin $A(2000) B R C A 1$ and $B R C A 2$ are necessary for the transcriptioncoupled repair of the oxidative 8-oxoguanine lesion in human cells. Cancer Res. 60: $5548-5552$
63. Bertrand P, Tishkoff DX, Filosi N, Dasgupta Rand Kolodner RD (1998) Physical interaction between components of DNA mismatch repair and nucleotide excision repair. Proc. Natl. Acad. Sci. USA 95: 14278-14283

64. Duckett DR, Drummond JT, Murchie AI, Reardon JT, Sancar A, Lilley DM and Modrich P (1996) Human MutSalpha recognizes damaged DNA base pairs containing O6-methylguanine, O4-methylthymine, or the cisplatin-d(GpG) adduct. Proc. Natl. Acad. Sci. USA 93: 6443-6447

65. Gowen LC, Avrutskaya AV, Latour AM, Koller BHand Leadon SA (1998)BRCA1 required for transcription-coupled repair of oxidative DNA damage. Science 281: $1009-1012$

66. Abbott DW, Thompson ME, Robinson-Benion C, Tomlinson G, Jensen RA and Holt JT (1999) BRCA1 expression restores radiation resistance in BRCA1defective cancer cells through enhancement of transcription-coupled DNA repair. J. Biol. Chem. 274: 18808-18812

67. Wang Y, Cortez D, Yazdi P, Neff N, Elledge SJ and Qin J (2000) BASC, a super complex of BRCA1-associated proteins involved in the recognition and repair of aberrant DNA structures. Genes Dev. 14: 927-939

68. Wang Q, Zhang H, Guerrette S, Chen J, Mazurek A, Wilson T, Slupianek A, Skorski T, Fishel R and Greene MI (2001) Adenosine nucleotide modulates the physical interaction between hMSH2 and BRCA1. Oncogene 20: 4640-4649

69. Bellacosa A, Cicchillitti L, Schepis F, Riccio A, Yeung AT, Matsumoto Y, Golemis EA, Genuardi M and Neri G (1999) MED1, a novel human methyl-CpGbinding endonuclease, interacts with the DNA mismatch repair protein MLH1. Proc. Natl. Acad. Sci. USA 96: 3969-3974

70. David SS and Williams SD (1998) Chemistry of glycosylases and endonucleases involved in base-excision repair. Chem. Rev. 98: 1221-1261

71. Hickson ID (1997) General introduction to DNA base excision repair. In Base Excision Repair of DNA Damage, Hickson ID, eds (Austin, Texas: Landes Bioscience (Springer-Verlag)) pp. 1-5

72. Krokan HE, Nilsen H, Skorpen F, Otterlei M and Slupphaug G (2000) Base excision repair of DNA in mammalian cells. FEBS Lett. 476: 73-77

73. Wallace SS (1994) DNA damages processed by base excision repair: biological consequences. Int. J. Radiat. Biol. 66: 579-589

74. Saparbaev M and Laval J (1999) Enzymology of the repair of etheno adducts in mammalian cells and in Escherichia coli. IARC Sci. Publ. 150: 249-261

75. Oda S, Humbert O, Fiumicino S, Bignami M and Karran P (2000) Efficient repair of $A / C$ mismatches in mouse cells deficient in long-patch mismatch repair. EMBO J. 19: 1711-1718

76. Neddermann P, Gallinari P, Lettieri T, Schmid D, Truong O, Hsuan JJ, Wiebauer Kand Jiricny J (1996) Cloning and expression of human G/T mismatch-specific thymine-DNA glycosylase. J. Biol. Chem. 271: 12767-12774

77. Bellacosa A (2001) Role of MED1 (MBD4) Gene in DNA repair and human cancer. J. Cell. Physiol. 187: 137-144

78. Petronzelli F, Riccio A, Markham GD, Seeholzer SH, Stoerker J, Genuardi M, Yeung AT, Matsumoto Y and Bellacosa A (2000) Biphasic Kinetics of the Human DNA Repair Protein MED1 (MBD4), a Mismatch-Specific DNA N-Glycosylase. J. Biol. Chem. 275: 32422-32429

79. Drummond JT and Bellacosa A (2001) Human DNA mismatch repair in vitro operates independently of methylation status at $\mathrm{CpG}$ sites. Nucleic Acids Res. 29: $2234-2243$

80. Hendrich B, Hardeland U, Ng HH, Jiricny J and Bird A (1999) The thymine glycosylase MBD4 can bind to the product of deamination at methylated $\mathrm{CpG}$ sites. Nature 401: $301-304$

81. Petronzelli F, Riccio A, Markham GD, Seeholzer SH, Genuardi M, Karbowski M, Yeung AT, Matsumoto $Y$ and Bellacosa A (2000) Investigation of the substrate spectrum of the human mismatch-specific DNA N-glycosylase MED1 (MBD4): Fundamental role of the catalytic domain. J. Cell. Physiol. 185: 473-480

82. Shen JC, Rideout WM and Jones PA (1994) The rate of hydrolytic deamination of 5-methylcytosine in double-stranded DNA. Nucleic Acids Res. 22: 972-976

83. Bader S, Walker M, Hendrich B, Bird A, Bird C, Hooper M and Wyllie A (1999) Somatic frameshift mutations in the MBD4 gene of sporadic colon cancers with mismatch repair deficiency. Oncogene 18: 8044-8047

84. Riccio A, Aaltonen LA, Godwin AK, Loukola A, Percesepe A, Salovaara R, Masciullo V, Genuardi M, Paravatou-Petsotas M, Bassi DE, Ruggeri BA, KleinSzanto AJP, Testa JR, Neri G and Bellacosa A (1999) The DNA repair gene MBD4 (MED1) is mutated in human carcinomas with microsatellite instability. Nature Genet. 23: 266-268 
85. Saparbaev M and Laval J (1998) 3,N $N^{4}$-ethenocytosine, a highly mutagenic adduct, is a primary substrate for Escherichia coli double-stranded uracil-DNA glycosylase and human mismatch-specific thymine-DNA glycosylase. Proc. Natl. Acad. Sci. USA 95: 8508-8513

86. Lieb M and Bhagwat AS (1996) Very short patch repair: reducing the cost of cytosine methylation. Mol. Microbiol. 20: 467-473

87. Stambuk S and Radman M (1998) Mechanism and control of interspecies recombination in Escherichia coli. I. Mismatch repair, methylation, recombination and replication functions. Genetics 150: 533-542

88. Evans $E$ and Alani E (2000) Roles for mismatch repair factors in regulating genetic recombination. Mol. Cell. Biol. 20: 7839-7844

89. Sugawara N, Paques F, Colaiacovo M and Haber JE (1997) Role of Saccharomyces cerevisiae Msh2 and Msh3 repair proteins in double-strand break-induced recombination. Proc. Natl. Acad. Sci. USA 94: 9214-9219

90. Myung K, Datta A, Chen C and Kolodner RD (2001) SGS1, the Saccharomyces cerevisiae homologue of BLM and WRN, suppresses genome instability and homeologous recombination. Nat. Genet. 27: 113-116

91. Rizki A and Lundblad V (2001) Defects in mismatch repair promote telomeraseindependent proliferation. Nature 411: 713-716

92. Langland G, Kordich J, Creaney J, Goss KH, Lillard-Wetherell K, Bebenek K, Kunkel TA and Groden J (2001) The Bloom's syndrome protein (BLM) interacts with $\mathrm{MLH} 1$ but is not required for DNA mismatch repair. J. Biol. Chem. 276: $30031-30035$

93. Ciotta C, Ceccotti S, Aquilina G, Humbert O, Palombo F, Jiricny J and Bignami M (1998) Increased somatic recombination in methylation tolerant human cells with defective DNA mismatch repair. J. Mol. Biol. 276: 705-719

94. de Wind N, Dekker M, Berns A, Radman M and te Riele H (1995) Inactivation of the mouse Msh2 gene results in mismatch repair deficiency, methylation tolerance, hyperrecombination, and predisposition to cancer. Cell 82:321-330

95. Roeder GS (1997) Meiotic chromosomes: it takes two to tango. Genes Dev. 11 2600-2621

96. Baker SM, Bronner CE, Zhang L, Plug AW, RobatzekM, Warren G, ElliottEA, Yu J, Ashley T, Arnheim N et al. (1995) Male mice defective in the DNA mismatch repair gene PMS2 exhibit abnormal chromosome synapsis in meiosis. Cell 82 : $309-319$

97. Baker SM, Plug AW, Prolla TA, Bronner CE, Harris AC, Yao X, Christie DM, Monell C, Arnheim N, Bradley A, Ashley T and Liskay RM (1996) Involvement of mouse Mlh1 in DNA mismatch repair and meiotic crossing over. Nat. Genet. 13: $336-342$

98. de Vries SS, BaartEB, Dekker M, Siezen A, de Rooij DG, de Boer P and te Riele $H$ (1999) Mouse MutS-like protein Msh5 is required for proper chromosome synapsis in male and female meiosis. Genes Dev. 13: 523-531

99. Kneitz B, Cohen PE, Avdievich E, Zhu L, Kane MF, Hou Jr H, Kolodner RD, Kucherlapati R, Pollard JW and Edelmann W (2000) MutS homolog 4 localization to meiotic chromosomes is required for chromosome pairing during meiosis in male and female mice. Genes Dev. 14: 1085- 1097

100. Nakagawa T, Datta A and Kolodner RD (1999) Multiple functions of MutS- and MutL-related heterocomplexes. Proc. Natl. Acad. Sci. USA 96: 14186-14188

101. Branch P, Aquilina G, Bignami M and Karran P (1993) Defective mismatch binding and a mutator phenotype in cells tolerant to DNA damage. Nature 362 : $652-654$

102. Kat A, Thilly WG, Fang W-H, Longley MJ, Li G-M and Modrich P (1993) An alkylation-tolerant, mutator human cell line is deficient in strand-specific mismatch repair. Proc. Natl. Acad. Sci. USA 90: 6424-6428

103. Hawn MT, Umar A, Carethers JM, Marra G, Kunkel TA, Boland CR and Koi M (1995) Evidence for a connection between the mismatch repair system and the G2 cell cycle checkpoint. Cancer Res. 55: $3721-3725$
104. Buermeyer AB, Wilson-Van Patten C, Baker SM and Liskay RM (1999) The human MLH1 cDNA complements DNA mismatch repair defects in Mlh1deficient mouse embryonic fibroblasts. Cancer Res. 59: 538-541

105. Karran P and Bignami M (1999) Mismatch repair and cancer. In DNA Recombination and Repair, Smith PJ and Jones CJ, eds (New York: Oxford Univ. Press) pp. 66-159

106. Zhang H, Richards B, Wilson T, Lloyd M, Cranston A, Thorburn A, Fishel R and Meuth M (1999) Apoptosis induced by overexpression of hMSH2 or hMLH1. Cancer Res. 59: $3021-3027$

107. Levine AJ (1997) p53, the cellular gatekeeper for growth and division. Cell 88 : $323-331$

108. Duckett DR, Bronstein SM, Taya Y and Modrich P (1999) hMutS $\alpha$ - and hMutL $\alpha$ dependent phosphorylation of p53 in response to DNA methylator damage. Proc. Natl. Acad. Sci. USA 96: $12384-12388$

109. Hickman MJ and Samson LD (1999) Role of DNA mismatch repair and p53 in signaling induction of apoptosis by alkylating agents. Proc. Natl. Acad. Sci. USA 96: $10764-10769$

110. Bunz F, Hwang PM, Torrance C, Waldman T, Zhang Y, Dillehay L, Williams J, Lengauer C, Kinzler KW and Vogelstein B (1999) Disruption of p53 in human cancer cells alters the responses to therapeutic agents. J. Clin. Invest. 104: 263-269

111. Oda K, Arakawa H, Tanaka T, Matsuda K, Tanikawa C, Mori T, Nishimori H, Tamai K, Tokino T, Nakamura Y and Taya Y (2000) p53AIP1, a potential mediator of p53-dependent apoptosis, and its regulation by Ser-46phosphorylated p53. Cell 102: 849-862

112. Nelson WG and Kastan MB (1994) DNA strand breaks: the DNA template alterations that trigger p53-dependent DNA damage response pathways. Mol. Cell. Biol. 14: 1815-1823

113. Fink D, Aebi S and Howell SB (1998) The role of DNA mismatch repair in drug resistance. Clin. Cancer Res. 4: 1-6

114. KharbandaS, RenR, PandeyP, Shafman TD, Feller SM, WeichselbaumRR and Kufe DW (1995) Activation of the c-Abl tyrosine kinase in the stress response to DNA-damaging agents. Nature 376: 785-788

115. Nehme A, Baskaran R, Aebi S, Fink D, Nebel S, Cenni B, Wang JY, Howell SB and Christen RD (1997) Differential induction of c-Jun NH2-terminal kinase and c-Abl kinase in DNA mismatch repair-proficient and -deficient cells exposed to cisplatin. Cancer Res. 57: 3253-3257

116. Gong JG, Costanzo A, Yang HQ, Melino G, Kaelin Jr WG, Levrero M and Wang JY (1999) The tyrosine kinase c-Abl regulates p73 in apoptotic response to cisplatin-induced DNA damage. Nature 399: 806-809

117. Zeng M, Narayanan L, Xu XS, Prolla TA, Liskay RM and Glazer PM (2000) lonizing radiation-induced apoptosis via separate Pms2- and p53-dependent pathways. Cancer Res. 60: 4889-4893

118. Toft NJ, Winton DJ, Kelly J, Howard LA, Dekker M, te Riele H, Arends MJ, Wyllie AH, Margison GP and Clarke AR (1999) Msh2 status modulates both apoptosis and mutation frequency in the murine small intestine. Proc. Natl. Acad. Sci. USA 96: $3911-3915$

119. Wu J, Gu L, Wang H, Geacintov NE and Li GM (1999) Mismatch repair processing of carcinogen-DNA adducts triggers apoptosis. Mol. Cell. Biol. 19: $8292-8301$

120. Ruschoff J, Wallinger S, Dietmaier W, Bocker T, Brockhoff G, Hofstadter F and Fishel R (1998) Aspirin suppresses the mutator phenotype associated with hereditary nonpolyposis colorectal cancer by genetic selection. Proc. Natl. Acad. Sci. USA 95: $11301-11306$ 